\title{
Non-representational thinking: Methodologische Überlegungen anhand des Bonner Sperrmülllassemblages
}

\author{
Erik Bertram \\ Geographisches Institut, Universität Bayreuth, Germany \\ Correspondence to: Erik Bertram (erik.bertram@uni-bayreuth.de)
}

Received: 29 March 2016 - Revised: 9 September 2016 - Accepted: 9 September 2016 - Published: 24 October 2016

\begin{abstract}
Kurzfassung. This paper undertakes a creative exploration of bulky rubbish [sperrmüll] in the City of Bonn by expanding on and utilising what has come to be known as non-representational thinking. Through a nonrepresentational engagement with sperrmüll, I shed light on waste as a significant human-nonhuman ecology as it appears in an especially routinised manifestation in urban space. In doing so, the paper contributes to a discussion about enacting non-representational methodologies within German-speaking human geography. Here, there are three methodological interventions for a non-representational endeavour: (1) interfering, (2) material thinking, and (3) writing and presentation. These interventions are supported by revisiting Deleuze and Guattari's notion of assemblage; using it as a way to attend to sperrmüll as an area-sized fabrication connecting humans and various other materialities, and which directs thought towards a wide array of agencies. In conclusion, nonrepresentational perspectives are advanced as a way to expose the affective ecologies that enable certain actions and hamper others.
\end{abstract}

\section{Einleitung}

Der Abfall in Bonn wird alle drei Monate zu etwas Andersartigem, zu etwas Ungewohnten. Viermal im Jahr stellen die Altstadtbewohner_innen ${ }^{1}$ gesammelt ihre ungewollten, sperrigen Gegenstände auf die Straße und lassen unbekannte Formen im öffentlichen Raum entstehen. Obwohl Sperrmüll als eine routinierte Form der Abfallentsorgung bekannt ist, und somit banal vorkommen mag, so wird er in der Bonner Altstadt für manche Menschen zu einem besonderen Ereignis. Für manche Menschen stellt er ein überraschendes Eingreifen in ihr Leben dar, das neue Bilder, Emotionen, Wünsche oder Bedürfnisse hervorruft. Wiederum andere sehen im Sperrmüll eine praktische Möglichkeit, etwas (für sie) Wertvolles und Nützliches zu finden. Während dieser Zeit ist Müll nicht mehr nur etwas, das es gilt loszuwerden. (Sperr-)Müll

\footnotetext{
${ }^{1}$ Die Altstadt ist ein Quartier in der Bonner Nordstadt. Sie bezeichnet eine Gegend, die durch viele Gründerzeithäuser, eine hohe Einwohnerdichte sowie eine hohe Nutzungsdurchmischung gekennzeichnet ist.
}

ist in diesen Momenten rätselhaft, anziehend oder nervig; jedenfalls ist er dann mehr als nur eine Ansammlung von überflüssig gewordenen Gegenständen. Er ist nicht bloß das Resultat menschlichen Tuns, sondern auch eine Aggregation von Dingen, die uns materiell herausfordert, die uns in die eine Richtung schiebt und wieder in eine andere zerrt. Aber warum über den Sperrmüll einer deutschen Stadt nachdenken?

Sarah A. Moores (2011) politisch-ökologische Analyse des globalen Müllproblems verleiht diesen Überlegungen eine drängende Relevanz. Sie beschreibt, wie Müll unseren Globus sowohl als Ware als auch als toxisches Nebenprodukt umrundet und so Überschneidungspunkte von unterschiedlichen sozialen, physischen und politischen Prozessen bildet, die eng mit globalen Kapitalflüssen und Prozessen ungleicher Entwicklung verzahnt sind sowie von ungleichen Machtbeziehungen tief durchdrungen sind. Müll könnte daher als die vielleicht fundamentalste vom Menschen initiierte Ökologie überhaupt bezeichnet werden (Peet et al., 2011:30). Eine zentrale Forderung von Moore (2011) ist daher, eben jene Knoten, die Müll ganz lokal bildet, auch örtlich sichtbar 
zu machen, wodurch Räume der Entsorgung wieder mit Räumen des Konsums in Beziehung gebracht werden können. Dadurch wird es möglich, der Konsumkultur des globalen Kapitalismus etwas entgegenzusetzen (ebd.:143).

Dafür kann sozialwissenschaftliche und geographische Forschung Müll nicht mehr nur als etwas Passives untersuchen. Das heißt als etwas, dass nur technisch kontrolliert werden muss (ebd.) oder dass die Logiken oder Unlogiken von Gesellschaften repräsentiert (Hawkins, 2006:2). Um den Verschränkungen von Müll besser begegnen zu können, muss Müll im Gegenteil auch in seiner Materialität konzeptualisiert werden (Gregson und Crang, 2010; Moore, 2012). Müll in seiner Materialität zu denken, bedeutet seine multiplen Potentiale zu adressieren, die nicht vollständig geordnet, kontrolliert oder repräsentiert werden können (Pikner und Jauhiainen, 2014:42; Edensor, 2005:316-317). Er ist vielmehr selbst aktiver Teil von unterschiedlichen Verbindungen und verändert dadurch konstant unsere sozio-materielle Welt (Gabrys, 2009). In der Humangeographie wird ein solches pluralistisches Verständnis von Materialität unter anderem von Bestrebungen voran getrieben, die im Zuge des material turns entstanden sind (Thrift und Dewsbury, 2000) und sich unter dem Label non-representational theory (Thrift, 2008) oder geographies (Anderson und Harrison, 2010) zusammenfassen lassen. Nicht-repräsentationale Arbeiten sind sehr divers und heterogen in ihren Theoriebezügen sowie Heranund Vorgehensweisen (ebd.), daher lassen sie sich vielleicht besser als ein Denkstil beschreiben. Non-representational thinking (NRT) hat zur zentralen Annahme, dass Menschen wichtige Akteure innerhalb der (sozialen) Welt sind. Sie können aber nicht als einzig aus sich heraus handelnde Wesen verstanden werden, sondern müssen auch innerhalb materieller Prozesse theoretisiert werden, die innerhalb und außerhalb des menschlichen Handelns und Repräsentierens operieren (Dewsbury et al., 2002).

Im anglophonen Kontext liegen die ersten nichtrepräsentationalen Arbeiten bereits etwas zurück ${ }^{2}$ und es gibt verschiedene Anregungen, wie methodisch vorgegangen werden kann. ${ }^{3}$ Trotzdem ist nicht-repräsentationales Arbeiten noch keine sozial- bzw. kulturwissenschaftliche Routineaufgabe geworden. Phillip Vannini (2015:2) hält fest, dass NRT zwar zurzeit wirkungsreich und viel beachtet ist, aber dennoch oftmals nur unzureichend verstanden wird. Das hängt auf der einen Seite mit den vielen verschiedenen Bezugspunkten von NRT und einer damit einhergehenden Ver-

\footnotetext{
${ }^{2}$ Hyden Lorimer (2015:179) sieht NRT 2016 in sein drittes Jahrzehnt eintreten.

${ }^{3}$ Für einen ersten Überblick siehe Davies und Dwyer (2007) oder Vannini (2015). Saunders (2013), Bates (2013) oder Waitt et al. (2014) bieten Beispiele, wie der klingende Überfluss des Lebens thematisiert werden kann. Für verschiedene Beispiele, wie Videos für nicht-repräsentationales Denken genutzt werden kann siehe Bates (2013), Lorimer (2010), Richardson-Ngwenya (2014), Simpson (2011, 2012, 2013), Oldrup und Carstensen (2012), Dirksmeier und Helbrecht (2013).
}

worrenheit zusammen. Auf der anderen Seite gab es bisher nur wenige klassisch empirisch-orientierte Anwendungsversuche und dadurch sind noch viele methodologische Fragen offen (ebd.).

Auch in den deutschsprachigen Geographien entstehen immer mehr Arbeiten unter bzw. in Abgrenzung zu dem Label nicht-repräsentationale Geographien (Kazig, 2007; Dirksmeier und Helbrecht, 2008, 2010; Korf, 2007, 2012; Strüver, 2011; Schurr, 2014; Hutta, 2015). Ebenfalls in Bezug auf die Politische Ökologie bzw. Politische Geographie sind die ersten Arbeiten entstanden, die mit nichtrepräsentationalen Gedanken in Verbindung stehen (Mattissek und Wiertz, 2014; Militz und Schurr, 2015; Müller, 2015). Allerdings gibt es aus der deutschsprachigen Wissenschaftsgemeinschaft, bis auf einige Ausnahmen (vgl. Dirksmeier und Helbrecht, 2013), kaum methodologische Beiträge zum nicht-repräsentationalen Arbeiten. Im deutschsprachigen Kontext ist daher eine vielleicht noch größere methodologische Lücke hinsichtlich einer nicht-repräsentationalen Denkweise zu sehen, als in dem, von Vannini adressierten, internationalen Diskurs.

Ein deutschsprachiger Beitrag ist daher aus mindestens drei Gründen lohnend: Erstens herrschen z.T. unterschiedliche Denkstile und Schreibweisen in den anglophonen und germanophonen Geographien vor (vgl. Hannah, 2016; Hesse, 2016; Jöns und Freytag, 2016). Selbstverständlich ist dies eine stark vereinfachte Unterscheidung zwischen zwei in sich verwobenen Wissenschaftslandschaften. Dennoch verstehe ich meinen Beitrag als Mashup zwischen Denk- und Schreibtraditionen: Er ist eine stärker aus der Empirie heraus gedachte (germanophone) Auseinandersetzung mit (anglophonen) theoriebetonteren nicht-repräsentationalen Gedanken. Zweitens gibt es in der deutschsprachigen Debatte rund um NRT eine dezidierte Hinwendung zu der Bezeichnung mehr-als-repräsentationalen Geographien (vgl. Schurr und Strüver, 2016) auf Kosten einer der wichtigsten politischen Potentiale von nicht-repräsentationalen Denken: ihr grundsätzliches Hinterfragen von Denkgewissheiten (vgl. Kapitel 2). Drittens sollte der sprachliche Unterschied, ob Sachverhalte auf Deutsch oder Englisch verhandelt werden, für die Vermittlung von Wissen (z.B. innerhalb von Debatten oder in der Lehre) nicht unterschätzt werden.

Im Zentrum dieses Artikels stehen zusammenfassend zwei in sich verschränkte Ziele. Erstens soll die vielleicht fundamentalste Ökologie, Müll, in einer seiner routinierten Dimensionen in den Blick genommen werden. Zweitens soll mit diesem Artikel ein deutschsprachiger Beitrag zu einer bisher hauptsächlich international geführten Methodologiedebatte geleistet werden. Dafür werde ich im Folgenden drei methodologische Ankerpunkte von non-representational thinking skizzieren: (1) Erschließen, (2) material thinking und (3) Schreiben und Präsentieren. Diese verdeutlichen, dass das Arbeiten mit und durch nicht-repräsentationale Ideen ein Experimentieren einfordert: ein Experimentieren sowohl beim Finden von Methoden, welche die Materialität des 
Untersuchungsprozesses betonen, als auch ein Experimentieren im Schreibprozess. Im Anschluss wird Gilles Deleuze und Félix Guattaris (bspw. 1992) Assemblagekonzept aufgegriffen, um genau dieses Experimentieren zu versuchen. Bei der Betrachtung des Sperrmülls als Assemblage wird offenkundig, dass man innerhalb des Sperrmülls mit eigenen sowie fremden Wertvorstellungen und Bedürfnissen konfrontiert wird und dass eine Handlung immer nur innerhalb eines Assemblages gedacht werden kann. Außerdem lässt sich innerhalb der Sperrmüllmaschine erleben, wie auch im globalen Norden Müll und Gerechtigkeit eng miteinander verzahnt sind. Daher zeigt ein nicht-repräsentationaler Denkstil, wie in unserem Alltag Möglichkeitsräume für andere Denk- und Handlungsweisen oder ,affective habit ecologies“ (Dewsbury, 2012) zu finden sind. Diese lassen sich allerdings nur durch ein In-Verbindung-Setzen innerhalb von Assemblages herausarbeiten.

\section{Non-representational thinking}

Non-representational thinking (NRT) ist der Versuch, die multisensorischen, präkognitiven, körperlichen, nichtdiskursiven Dimensionen des menschlichen Lebens im Forschungsprozess zu berücksichtigen bzw. eben diese zu untersuchen (Lorimer, 2005). Für diese Art des Arbeitens hat sich auch die Begriffskombination more-than-representational eingebürgert. Laut Lorimer (ebd.:84) hat sich das Präfix „non“ als eine unglückliche Hürde herausgestellt, wohingegen das ,more-than“ die Ideen von NRT einem breiteren ,geographischen Publikum“ zugänglich machen soll. Dewsbury (2010:155) warnt jedoch vor dem angeblich offeneren Terminus. Die Gefahr des „,mehr-als“ ist, dass man wieder in ,repräsentationale Systeme hineinrutscht“, welche behaupten, Authentizitäten oder universelle Wahrheiten zu produzieren (Anderson und Harrison, 2010:19). Die Vorsilbe „mehr-als“ verweist daher eher auf die Frage, was zählt (biotisch, abiotisch, Menschen, Tiere, Bakterien etc.), wohingegen die Vorsilbe ,nicht“ grundsätzlichere Gewissheiten infrage stellen möchte. Daher soll nicht-repräsentational hier als eine Art zu denken verstanden werden, welche das „dogmatische Bild von dem, was als Gedanke zählt" hinterfragt (Thrift, 2004:81; meine Übersetzung). Dementsprechend verbleibe ich in meinem Beitrag auch bei dem Begriffspaar nicht-repräsentational.

Dieser Anspruch Denkgewissheiten zu hinterfragen bedeutet, dass für jede Forschungsfrage ein individueller $\mathrm{Zu}$ gang gefunden werden muss, der die jeweilige Persönlichkeit im Forschungskontext berücksichtigt (vgl. Dewsbury, 2009). Für meine eigene Erkundung des Sperrmülls habe ich drei temporale methodologische Etappen herausgefiltert, die den weiteren Verlauf dieses Kapitels strukturieren werden und die ich wie folgt beschreibe: (1) zuvor, (2) im Mittelpunkt des Geschehens und (3) Weiterwirken. In Mitten dieser drei Phasen des Forschungsablaufs werden sich zeitgleich drei wichtige nicht-repräsentationale methodologische Ankerpunkte zeigen: (1) Erschließen, (2) material thinking und (3) Schreiben und Präsentieren. Wie ich deutlich machen werde, überlagern sich diese drei schematisch aufgeführten zeitlichen und vollziehenden Dimensionen des Forschungsablaufs. Überlagerung ist auch der Einstieg zu den nun folgenden methodologischen Überlegungen.

\subsection{Zuvor: Erschließen}

John Law (2004:2) postuliert zu Beginn seines Buches After method: ,[S]imple clear descriptions don't work if what they are describing is not itself very coherent. The very attempt to be clear simply increases the mess". Wenn man also versuchen möchte, die Welt in ihrer Unbeständigkeit, Unbestimmtheit und in ihren unregelmäßigen Singularitäten zu denken, dann erweist sich die starre Anwendung von Methoden und Ideen nicht als hilfreich (ebd.:4). Stattdessen braucht es dafür ein anderes Verständnis von sozialwissenschaftlichen Methoden. Für Law (ebd.:67) - rekurrierend auf Mol (1999) und beeinflusst von Latours Akteur-NetzwerkTheorie - gilt es Methodologie als „ontologische Politik“ zu verstehen. Forschung produziert mithilfe von bestimmten Methoden und innerhalb eines spezifischen materiellen Settings (Labor, Ratten, Büchern, Interviewpartner_innen, Ideen, scientific communities, Diagrammen, Daten, Bakterien, Gebäuden, dem Aktienmarkt, Sandwiches, Stiften, Konversationen, Computern, Maschinen usw.) bestimmte Realitäten. Forschung ist somit ein Akt des Eingreifens, der gewisse Wirklichkeiten hervorbringt und andere ausschließt (ebd.). Diese Einsicht ist auch für das nicht-repräsentationale Arbeiten zentral. Denn sie beinhaltet, dass die Umstände von (sozial)wissenschaftlicher Wissensproduktion auch Teil des Forschungsergebnis sind (Dewsbury, 2009:323-324) (siehe Unterkap. 2.2). Zudem steckt in dieser Erkenntnis ein großes Potential. Sie ermöglicht die Chance, Forschung als Möglichkeit zu verstehen, neue Eintrittspunkte zum Alltagsleben zu schaffen (ebd.). Diese kreative Sichtweise spiegelt sich auch in einer affirmativen Grundannahme nichtrepräsentationalen Denkens wider. Affirmativ bedeutet hier, das Leben als Potential und den Prozess als Grundzustand zu verstehen. Wo die nächste Verbindung ist, ist dabei stets eine zentrale Frage (Anderson and Harrison, 2010).

Diesem affirmativen Blick auf das Leben nachzukommen, bedeutet für eine nicht-repräsentationale Denkweise, dass Theorie beweglich (abstrakt) bleiben muss, um sich den verschiedenen (virtuellen und aktualen) Möglichkeiten anzupassen (ebd.:8). Das hat natürlich auch epistemologische Konsequenzen. Eine nicht-repräsentationale Denkweise ist der Versuch, Vielheiten oder „Differenzen“ und ihre wechselseitigen Wiederholungen zu erschaffen (Thrift, 2004:83). Die Aufgabe von NRT ist dementsprechend auch, die Welt zu präsentieren und nicht zu versuchen, sie zu repräsentieren oder sie zu erklären (Dewsbury et al., 2002:438). Dies bedeutet allerdings nicht, dass Erklärungen nicht mehr Teil des akademi- 
schen Arbeitens sein sollen (Dewsbury, 2014:151). Vielmehr fordert NRT uns dazu auf, neue Formen des wissenschaftlichen Weltenerkundens zu finden. Dafür können die bildenden sowie darstellenden Künste oder andere Formen des kreativen Schaffens zurate gezogen werden, um das zu erweitern, was Bedeutung gegeben wird. Non-representational thinking bedeutet also, sich einem gewissen Experimentalismus zu öffnen (Dewsbury et al., 2002:439-440).

\subsection{Im Mittelpunkt des Geschehens: material thinking}

Wie oben bereits angedeutet folg der Einsicht, dass der eigene Forschungsschauplatz ein unübersichtliches Feld ist, welches von komplexen Überschneidungen von Linien (intellektuelle oder disziplinäre Grenzen, Theorien, Trends, Finanzierungen, praktische Hürden, politische Restriktionen, materielle Schranken usw.) geprägt ist, eine weitere Erkenntnis: Ich muss nicht nur meine eigenen Wege durch diese komplexen Überschneidungen finden, sondern ich stecke körperlich in ihnen fest (Dewsbury und Naylor, 2002:256). Performative Vorgehensweisen messen daher den Interferenzen zwischen der materiellen Dimension der Forschungsobjekte/räume und dem Körper der/des Stakeholder_in eine große Bedeutung bei. Der Forschungsprozess hängt immer untrennbar mit der Person zusammen, die diesen durchläuft (ebd.). Diese einfache und auch schon in anderen Forschungsdisziplinen (meist von feministischen oder postkolonialen Theorien beeinflusste) weit verbreitete Einsicht geht für eine nicht-repräsentationale Denkweise mit zwei wichtigen Konsequenzen einher (ebd.:256-257; Dewsbury et al., 2002:439): (1) Unsere Körper haben ihre eigenen Handlungsweisen und wirken für sich sowie durch sich. Allerdings können wir versuchen, uns ihre Eigenheiten zu Nutze zu machen und sie gezielt als „Forschungswerkzeuge“ einzusetzen. (2) Affekte und Emotionen beeinflussen nicht nur unsere Forschungserfahrung, sondern sind auch gleichermaßen Teil unserer Einsichten. Sie ermöglichen uns, in bestimmter Weise zu handeln und nicht in anderen. Sie wirken sowohl ermöglichend als auch beschränkend. Nicht-repräsentationales Arbeiten ist dementsprechend immer ein Arbeiten mit und durch den Körper (Dewsbury, 2009). Das Denken durch bestimmte Objekte, Räume oder Materialitäten ermöglicht das Eintauchen in Ereignisse. Es sind Versuche, wie Dewsbury (2009:327; meine Hervorhebung) es so inspirierend beschreibt, „to gather a portfolio of ethnographic ,exposures“ that can act as lightning rods for thought". Es ist die Hoffnung, sich in einem Ort/einer Materialität zu verhaken, sich im Erfahren/Erleben eines Textes, eines Platzes, eines Geschehens, eines Steines, eines Rituals, einer Krankheit, einer Plastiktüte, eines Handys, einer Praktik etc. zu verlieren und affiziert zu werden. Aber bei diesem ,materiellen Denken“ (Thrift, 2008:8; Henare et al., 2007; Gabrys et al., 2013), gilt es stets zu beachten, dass es immer nur durch die „Sensation“ von Räumlichkeiten/Materialitäten erreicht werden kann. Diese ist immer zugleich materiell und immate- riell, zugleich menschlich und nicht-menschlich bzw. organisch und anorganisch (Dewsbury, 2009:326). Es gilt also, ein Experimentieren zu wagen, in dem wir unsere Aufmerksamkeit auch auf ,,das Einsetzende; auf das schlecht Geformte; das augenscheinlich Unerhebliche; das flüchtig Gefühlte; den Wunsch, der den Raum erhellt; die Abzweigungen, die wir nicht genommen haben (aber uns immer noch verfolgen) oder die Angst, die nächste Aufgabe zu erledigen“" lenken (Dewsbury et al., 2002:439, meine Übersetzung).

Methodisch spielt beim Erkunden von Affekten und Atmosphären von Städten bzw. gebauter Umwelt daher oft eine Mischung von ethnographischen Ansätzen (häufig teilnehmende Beobachtung) kombiniert mit kurzen Interviews, "Walk-Alongs" oder Fotodokumentationen, eine wichtige Rolle (Latham und McCormack, 2009; Miller, 2012; Roberts, 2012; Yi'En, 2013). Wobei nicht-repräsentationale Forschung darauf abzielt, die „teilnehmende Beobachtung“ zu einer ,beobachtenden Teilnahme“" zu verkehren (Dewsbury, 2009:327). Nicht-repräsentationale Anwendungen ethnographischer Methoden versuchen also eine gewisse zurückhaltende und beaufsichtigende Verhaltensweise abzulegen und sich stattdessen beherzt in die Situation zu begeben, zu interagieren und den Moment mit seinem ganzen Körper mitzuerleben (Thrift, 2008:109-150). Denn einen Moment zu erleben ist immer nur durch die eigene Involviertheit möglich, immer nur durch das eigene Werden denkbar. In diesem Sinne sind nicht-repräsentationale Ethnographien auch immer Autoethnographien (Reed-Danahay, 1997), in dem das Selbst, das Soziale und Materielle miteinander verschmilzt und neugeschrieben wird. In meinem Falle habe ich versucht, mich in die Situationen des Herausstellens und Abtransports des Sperrmülls durch die psychogeographische Methode des Umherschweifens [dérive] fallen zu lassen.

Gehen wird schon lange als erfolgreiche Methode gesehen, unsere alltäglichen Geographien der Städte und uns in ihnen zu erkunden (bspw. Michel de Certeau, 1988 oder Walter Benjamin, 2002). Das psychogeographische Konzept des Dérives ist dabei eine Technik, die es erlaubt, auf ungewohnte Weise die Stadt neu zu erkunden. Psychogeogaphie entstand aus der Bewegung der Situationisten der 1950/60er Jahre. Ihre Bewegung ist ein Versuch, ein Lebensmodell zu entwerfen, das sich der kapitalistischen Art und Weise die Stadt zu nutzen, diese zu denken und ihr Bedeutung zu verleihen offen entgegenstellt (Bassett, 2004; MacFarlane, 2005). Gehen spielt für die Psychogeograph_innen dabei eine wichtige Rolle. Beim situationistischen Gehen gilt es, den Zufall hier verstanden als Beliebigkeit - als bestimmendes Element zu vermeiden. Debord (1958) befürchtet, dass man beim beliebigen Umherwandern nicht loslassen kann und sich daher auch nicht treiben lässt. Anstelle dessen folgt man einem gewissen ,psychogeographische[n] Bodenprofil mit beständigen Strömen, festen Punkten und Strudeln“ (Debord, 1958, o.S.), die ein Neuentdecken der Stadt verhindern. Deswegen bedarf es aus Sicht der Situationisten gewisser experimenteller Techniken. Sie nutzen beispielsweise selbstausgedachte 
Algorithmen (z.B. 1. Möglichkeit links gehen, 2. rechts gehen, 2. links usw.) oder zeichnen mithilfe eines Gegenstandes (z.B. einem Wasserglas) Formen auf eine Stadtkarte und versuchen anschließend die eingezeichnete Strecke nachzulaufen (Bassett, 2004). Während man umherschweift, geben sich die Umherschweifenden „,den Anregungen des Geländes und den ihm entsprechenden Begegnungen" hin (Debord, 1958, o.S.). Durch dieses verspielte Konstruieren fördert das Umherschweifen ein Verhalten, das Menschen für andere psychogeographische Effekte der Stadt öffnet und sich dadurch von einem normalen Spaziergang unterscheidet (ebd.). Durch die Intentionslosigkeit eines Dérives - so die Hoffnung - eröffnen sich Durchbrüche zu bisher verdeckten Meinungen und Geschichten einer Stadt; der Dérive gewährt Einlass in das Kleine einer Stadt, in ihre Spalten und Risse, in ihre Unregelmäßigkeiten und deren Muster (Bassett, 2004; Jenks und Neves, 2000; Wrights \& Sites, 2006).

Wohingegen die Situationisten den Zufall kritisch sehen, bedarf es für das Gehen durch die Altstadt, während der Sperrmüll auf den Straßen steht, keiner besonderen Algorithmen. Der Müll sorgt dafür, dass Wahrnehmungs- und Bewegungsgewohnheiten aufgebrochen werden (vgl. DeSilvey, 2006; Edensor, 2005). Gehen ist in diesen Momenten also weder eine zielgerichtete Praktik, die jemanden von A nach B bringen soll, noch ein striktes psychogeographisches Verfahren. Es ist weder utilitaristisch noch ziellos. Walking ethnography ist beim Erkunden des Sperrmülls in der Altstadt ein Prozess von Anziehung und Ablenkung, von Orientierung und Desorientierung oder Kontinuität und Diskontinuität. Das Gehen durch die Altstadt ist dann ein Modus der Erkundung, ähnlich der Erfahrung, wie sie Peter Merriman (2005:154) für das Fahren auf der $M l$ beschreibt: Es eröffnet uns Einblicke in die ,complex habitations, practices of dwelling, embodied relations, material presences, placings and hybrid subjectivities associated with movement through such spaces“.

\subsection{Weiterwirken: Schreiben und Präsentieren}

Allen Grundintentionen von non-representational thinking zum Trotz müssen wissenschaftliche Untersuchungen immer auch verschriftlicht und präsentiert werden. Allerdings lässt sich die Sorge um den Schreibprozess auch ins Gegenteil verkehren. Schreiben wird dann als ein Prozess verstanden, der uns die Handlungen und Orientierungen, die unsere Geist-Körper in Reaktion auf die sozio-materiellen Umwelten vollziehen, zugänglicher macht. Der Schreibprozess ist ein Denk-, Erfahrungs- und Konstitutionsprozess. Selbstverständlich ist Schreiben immer schon eine Methode in sich selbst gewesen (DeLyser, 2009). Allerdings wird die Aufmerksamkeit oft auf einen Reflexionsprozess verengt. Das bedeutet nicht, dass die Reflexion von sozialen Positionen, Machtpotentialen, kulturellen Normen etc. nicht mehr erforderlich ist. Denn, wie es Dydia DeLyser (2009:352) formuliert, ,writing, as a personal and conscious process of langua- ge crafting, is embedded with issues of powers, politics, and ideology“. Allerdings ist der Schreibprozess auch eine affektive Erfahrung für den Schreibenden sowie später für den Lesenden (Deleuze und Guattari, 1996:191-237). Er kann als „experience of thought itself“" verstanden werden (Dewsbury, 2014:149). Den akademischen Schreibprozess als eine produzierende Aufgabe zu verstehen, kann daher auch ein befreiender Gedanke sein.

Für eine nicht-repräsentationale Art, die Dinge zu erkunden, ist das Fragenstellen und das Erarbeiten von unterschiedlichen Möglichkeiten wichtiger als das Finden von finalen - und somit äußerst temporären - Antworten. Daher versucht NRT auch nicht, empirische Beweise für die Richtigkeit einer Theorie zu sammeln, sondern Empirie als ein Voranschreiten im Denken zu nutzen. Das gleiche gilt für die Theorie. Theorie soll nicht (nur) empirische Belege widerspiegeln, sondern soll unsere Art, die Welt wahrzunehmen und zu denken verändern. Daraus folgt auch, dass empirisches Arbeiten immer zugleich auch theoretisches Arbeiten ist und umgekehrt (ebd.:324).

Non-representational writing ist folglich der Versuch, andere Formen des Schreibens auszutesten und dadurch das Denken in andere Richtungen zu lenken (vgl. Vannini, 2015). Eine wichtige Technik zu diesem Zweck ist, schreibend die kleinen Details unseres Alltagslebens in den Blick zu nehmen. Latham und McCormack (2009) schlagen dafür vor, sich die Arbeiten des Autors Georges Perec und seinen Schreibstil anzuschauen. Perec untersucht mit großen Mühen die kleinen Nuancen des Alltags. An einer Stelle in seinem Buch Species of spaces and other pieces (2008:210211) schreibt Perec Folgendes:

We live, true, we breathe, true; we walk, we open doors, we go down staircases, we sit at a table in order to eat, we lie down on a bed in order to sleep. How? Where? When? Why?

Describe your street. Describe another street. Compare.

Make an inventory of your pockets, of your bag. Ask yourself about the provenance, the use, what will become of each of the objects you take out.

Question your tea spoons. ${ }^{4}$

Das Lesen von Perec oder anderen Literat_innen (hilfreich für mich sind bspw. Cortázar, 2010; Federman, 1976; Foer, 2006) ermöglicht auf der einen Seite Anregungen für den eigenen Schreibstil zu erhalten. Auf der anderen Seite ist es eine Möglichkeit, wie Claire Colebrook (2014) argumentiert, eine inhumane Perspektive einzunehmen, in der die Literatur die Komplexität der erfahrenen Sensationen maximiert und gleichzeitig einen Weg findet, diese als solche Sensationserweiterungen zu präsentieren. Zudem geht es bei der

\footnotetext{
${ }^{4}$ Die zitierten Zeilen sind nicht in der dt. Übersetzung, Träume von Räumen (2013), enthalten. Daher zitiere ich hier die englische Fassung.
} 
Konsultation von Literatur um ein ständiges Verbindungssuchen, was auch für andere Kulturprodukte (Gemälde, Gedichte, Lieder, Installationen, Dokumentarfilme, Kinofilme, Podcasts etc.) gilt. Auf diese Weise verschmelzen die zeitlichen Abläufe einer Forschung. Selbst hinterher kann man sich noch im Mittelpunkt des Geschehens bzw. in der Phase des vorherigen Erschließens befinden.

Zusammenfassend lässt sich sagen, dass NRT Lücken akzeptiert, beispielsweise die Lücken zwischen dem, was wir erfahren und dem, was wir erfahren; zwischen dem, was wir erfahren und dem, was wir ausdrücken können; zwischen dem, was wir ausdrücken können und dem, was wir ausdrücken könnten; und zwischen dem, was wir schlussendlich ausdrücken und dem, was dadurch außen vor gelassen wird (Dewsbury, 2011b). NRT will die Da-zwischen (inbetweens), die Noch-ausstehenden (yet-to-comes), die Undaber (and, buts) (Doel, 2010:128), die Virtualitäten und Vitalitäten der Dinge in den Blick nehmen; es fordert uns dazu auf, Räume zwischen den Wörtern zu öffnen, die über die unmittelbaren Sinnesempfindungen hinausreichen (Dewsbury et al., 2002:439). Die Konfrontation mit dem Sperrmüll soll daher hier durch Deleuze und Guattaris (bspw. 1992:1142) Prinzip der Mannigfaltigkeit, der Vielheit und der Relationen gesucht werden. Es soll nicht darum gehen, was der Sperrmüll ist, sondern wie er sich verbindet: Es geht um die „und ... und ... und ...“ des Sperrmülls (ebd.:41). Für diese methodologische Prämisse bedarf es eines kurzen theoretischen Rückgriffs auf Deleuze und Guattaris Konzepts des Gefüges.

\section{Deleuze und Guattaris Konzept vom Gefüge}

Deleuze und Guattaris Konzept des Gefüges ${ }^{5}$ wird seit einigen Jahren in der englischsprachigen Humangeographie verhandelt (Robbins und Marks, 2009) und auch zunehmend in deutschsprachigen Publikationen rezipiert (Mattissek und Wiertz, 2014; Miggelbrink, 2014). Obwohl es in den unterschiedlichen humangeographischen Teilbereichen verwendet wird (z.B. Stadtgeographien, postkoloniale Geographien, Wirtschaftsgeographie u.v.a.), gibt es dennoch nicht das eine Verständnis bzw. die eine richtige Art der Anwendung dieses Konzepts (Anderson und McFarlane, 2011:124). Ganz allge-

\footnotetext{
${ }^{5}$ Deleuze und Guattari (1976) führen ihre Idee vom Gefüge oder von der Verkettung [Fr. agencement] erstmals in ihrem Buch Kafka: Für eine kleine Literatur ein. Oftmals wird auch in der deutschsprachigen Debatte die englische Übersetzung assemblage genutzt (vgl. Mattissek und Wiertz, 2014; Miggelbrink, 2014), um an internationale Theoriediskussionen anzuknüpfen (Mattissek und Wiertz, 2014). Für eine kritische Auseinandersetzung mit der englischen Übersetzung siehe z.B. Alliez und Goffey (2011:10-11). Das französische Wort betont viel stärker die Handlungsmacht eines Gefüges (ebd.). Im Folgenden werde ich das deutsche „Gefüge“ und das englische ,assemblage“ synonym verwenden. Auf „Verkettung“ werde ich allerdings bewusst verzichten, da es für mich eine gewisse Linearität suggeriert.
}

mein gesprochen betont die Assemblagedenkweise das $\mathrm{Zu}-$ sammenkommen von unterschiedlichen Elementen (McFarlane, 2011:652). Im Folgenden werde ich mich auf Deleuze und Guattaris Verständnis vom Gefüge beziehen.

Der Deleuze-Guattari'schen Konzeptualisierung vom Gefüge liegt ein neo-vitalistisches Verständnis der Welt zu Grunde. Das heißt, die Welt besteht aus Verbindungen, die konstant durch die kreative Kraft des Lebens entstehen. Diese Kraft nennen Deleuze und Guattari (1976, 1977, 1992, 1996) u.a. auch Wunsch/Verlangen/Begehren [Fr. désire] oder Wunschproduktion und sie ist der inhärente Antreiber von Gefügen. Die Verbindung zwischen beiden Konzepten ist elementar und das eine kann nicht ohne das andere gedacht werden (vgl. Halsey, 2005; Lash, 2006). Für das Denken mit und durch das Deleuze-Guattari'sche Assemblagekonzept ist es hilfreich, vier zentrale Aspekte des Konzeptes herauszustellen (Dewsbury, 2011a). (1) Gefüge müssen immer in ihrer Mannigfaltigkeit und Flüchtigkeit gedacht werden. Assemblages betonen die räumlichen und zeitlichen Veränderungen. Eine unbestimmte Anzahl von Elementen (Körper) ergeben ein Gefüge bzw. verbinden sich mit einem anderen Gefüge. Sie halten zusammen, sie bleiben bestehen, nur um in einem anderen Moment wieder auseinanderzutreiben. Deleuze (1988b:127) schreibt in seinem Buch über Spinoza: ,,[A] body can be anything, it can be an animal, a body of sounds, a mind, an idea, it can be a linguistic corpus, a social body, a collectivity“. Ein Körper wird durch zwei Dimensionen bestimmt: Erstens durch das kinetische Zusammenspiel der verschiedenen Elemente, aus dem er besteht: Geschwindigkeit und Langsamkeit, Bewegung und Rast. Zweitens wird ein Körper durch die dynamischen Kapazitäten bestimmt, also durch seine Fähigkeiten, andere Körper zu beeinflussen (zu affizieren) oder von anderen Körpern beeinflusst (affiziert) zu werden (ebd.:123). Daher ergibt sich (2) beim Denken durch und mit Assemblages ein starker Fokus auf Instabilitäten, Brüche oder Heterogenitäten. (3) Innerhalb eines Gefüges ist Soziales untrennbar mit Materiellem verwoben. Das bedeutet, dass Handlungsmacht nicht von einer Person, einem Element, ausgeht, sondern dass sie über die verschiedenen Körper, die ein Assemblage ergeben, verteilt ist. (4) Das Gefüge hat nur sich selbst zum Objekt, sich selbst und die unzähligen Verbindungen, die es mit anderen Gefügen herstellt. Das Gefüge ist der Versuch alles auf einer Ebene zu denken: das Subjekt, die Gemeinschaft, das Ereignis. Es kann daher auch innerhalb eines Gefüges keinen Unterschied zwischen Aktualisiertem und Nichtaktualisiertem geben (Anderson und Harrison, 2010:13Fn7). Ein Gefüge hat, vereinfacht gesagt, eine aktuale und eine virtuelle Komponente. Die aktuale Seite bezieht sich auf alles, was sich bereits manifestiert hat, z.B. in Handlungen, Gefühlen, Reaktionen oder Schmerzen. Die virtuelle Dimension deutet auf den Überfluss des Lebens hin, der sich noch nicht in konkreten Phänomenen aktualisiert hat.

Das Gefügekonzept bedeutet daher auch, dass es selbst nicht als statischer Terminus verstanden werden darf, son- 
dern vielmehr als ein ,process of putting together, of arranging and organising the compound of analytical encounters and relations" (Dewsbury, 2011a:150). Der Agencementgedanke beinhaltet stets, sich zu verbinden - auch konzeptionell. Kein Konzept kann bei Deleuze (und Guattari) für sich gedacht werden. ,[T] $[$ here is an almost circular quality to Deleuze's work: once you understand one term you can understand them all; but you also seem to need to understand all the terms to even begin to understand one" (Colebrook, 2002:xviii-ix). Was das Gefüge angeht, so ist es beispielsweise mit Konzepten wie Affekt, Werden, Rhizom oder Ereignis verbunden (Deleuze und Guattari, 1992). Das Assemblagekonzept betont also insbesondere die Exterioritäten. Ein Gefüge ist immer anschlussfähig. Jedes Assemblage hat ein Außen, welches eine Veränderung herbeiführt: Es befindet sich im stetigen Werden ${ }^{6}$. Das Werden unterstreicht die Interaktion zwischen den einzelnen Komponenten, die ein Assemblage formen, und vernachlässigt dabei bewusst die Eigenschaften der einzelnen Komponenten.

\begin{abstract}
„Man frage nie, was (...) [es] sagen will, ob es nun Signifikat oder Signifikant ist; man soll in (...) [ihm] nicht etwas verstehen, sondern sich viel mehr fragen, womit es funktioniert, in Verbindung mit was es Intensitäten eindringen läßt oder nicht, in welche Mannigfaltigkeit es seine eigene einführt und verwandelt, mit welchen organlosen Körpern es seinen eigenen konvergieren läßt. (...) [Es] existiert nur durch das und in dem, was ihm äußerlich ist. (...) [Es] ist selber also eine kleine Maschine (...)“ (Deleuze und Guattari, 1992:13).
\end{abstract}

Die bisher genannten Aspekte spiegeln sich im Kernpunkt des Deleuze-Guattari'schen Gefügekonzepts wider: der Tetravalenz. „Es gibt eine Tetravalenz des Gefüges: (1) Inhalt und Ausdruck; (2) Territorialität und Deterritorialisierung" (Deleuze und Guattari, 1992:699-700). Deleuze und Guattari (1992:123-124), beschreiben diese folgendermaßen: Der Inhalt steht für das reagierende Maschinengefüge der Körper, Aktionen und Passionen; der Ausdruck steht für das kollektive Äußerungsgefüge von Handlungen und Aussagen; Territorialität für die Artikulationslinie, und Deterritorialisierung für die Fluchtlinie. Dewsbury (2011a:150) findet für diese Vierwertigkeit ein Beispiel aus dem Alltag: fahrradfahrende Körper. Zwei Körper treffen aufeinander und bilden ein Gefüge: ein menschlicher Körper und der Fahrradkörper. Gemeinsam entwickeln sie neue Modi der Bewegung und der sozialen Interaktion. Der fahrradfahrende Körper verbessert

\footnotetext{
${ }^{6}$ Deleuze und Guattari (1992:371; Hervorhebung im Original) beschreiben den Prozess des Werdens wie folgt: „Werden heißt, ausgehend von Formen, die man hat, vom Subjekt, das man ist, von Organen, die man besitzt, oder von Funktionen, die man erfüllt, Partikel herauszulösen, zwischen denen man Beziehungen von Bewegung und Ruhe, Schnelligkeit und Langsamkeit herstellt, die dem, was man wird und wodurch man wird, am nächsten sind. In diesem Sinne ist das Werden der Prozeß des Begehrens.“
}

seine Fahrradfahrfähigkeiten; sein semiotisches und materielles Wissen, z.B. das Wissen um bestimmte Straßenverläufe, wird in dieses Assemblage hineingeschrieben und manifestiert sich. Dadurch entsteht eine Artikulationslinie bzw. ein neues Territorium. Nur durch das Zusammenkommen, die Flüsse zwischen diesen Gefügen, entsteht diese individuelle Handlung des Fahrradfahrens. Zahnräder drehen sich, Beine bewegen sich auf und ab, Räder rollen über den Asphalt. Es gibt immer ein Außen zu jedem Gefüge. Weder der menschliche Körper noch das Fahrrad können sich ohne den anderen in diesem Moment bewegen. Es sind diese Diesheiten („Haecceïtas“), die das Denken durch Gefüge herausarbeitet. Diesheiten, das sind Momente der Individuation, der Metamorphose von Dingen und Subjekten (Deleuze and Guattari, 1992:355). Daher kann ein Gefüge auch nie nur das unmittelbar Vorhandene sein, sondern es ist immer mit dem Virtuellen verbunden. Virtualität ist gleichbedeutend mit der ganzen Zeit oder durée [Dauer], wie Deleuze (1988a) sie nach Bergson nennt. Das bedeutet, dass ein Assemblage in seiner Kurzlebigkeit immer mit der ganzen Dauer verbunden ist. Es ist mit einer Dauerhaftigkeit verbunden - egal wie lange es Bestand hat (Deleuze und Guattari, 1996). Im Sinne einer neu-materialistischen (spekulativ-realistischen) Untersuchung von Alltagsgefügen (z.B. Roberts, 2012) werde ich versuchen, über das unvorhersehbare Werden zu spekulieren das inmitten der allgegenwärtigen Choreographie der affektiven Kapazitäten von Sperrmüll hervortritt. Ich will uns gleich mitten in das Sperrmüllassemblage setzen, schließlich ist die Mitte der richtige Ort für den Anfang (Deleuze und Guattari, 1992:41-42).

\section{$4 \quad$ Assemblierung des Sperrmülls}

Obwohl ich die Materialität des Sperrmüllassemblages in den Blick nehmen will, bleiben menschliche Akteure auch immer Teil des Ganzen. Denn wenn ich durch die Straßen laufe, dann sehe ich Menschen, die den Sperrmüll herausstellen, die ihn durchstöbern, die Gegenstände aufnehmen, an anderer Stelle wieder ablegen oder mitnehmen. Die Körper fügen sich zusammen. Um eine materielle Denkweise einzunehmen, ist es notwendig, sich von einem linearen, hierarchischen und subjekt-zentrierten Verständnis von Handlungsfreiheit (agency) zu lösen. Es ist ein Versuchen, sich den Dingen mit einem Agencyverständnis zu nähern, in dem Denkkategorien wie Ursache (cause) oder Wirkung (effect) miteinander verschmolzen sind (Bennett, 2010:33). Alles hängt beim Sperrmüllen mit allem zusammen, aber nichts passiert in direkter, linearer Abstimmung zueinander. Es geht um eine gewisse „emergent causality“ (ebd.) innerhalb von Assemblages. Diese Assemblagekausalität beinhaltet drei Aspekte: (1) Eine Bewegung auf einer Ebene hat Auswirkungen auf eine andere Ebene. Allerdings kann im Vorfeld nicht genau festgestellt werden, was die Bewegung ist, bis nicht auch ihre Effekte erkennbar sind. (2) Die neu 
entstandenen Effekte werden auf der zweiten Ebene aufgenommen, sodass es nur eine verzerrte Verbindung zwischen der ursprünglichen Bewegung und den ausgelösten Effekten gibt. (3) Es gibt Serien von unvorhersehbaren Feedbackloops, welche die Resultate der Austauschbewegungen zwischen der ersten und zweiten Ebene stabilisieren. „The new emergent is shaped not only by external forces that become infused into it but also by its own previously under-tapped capacities for reception and self-organization" (Connolly, 2004:342-343 zitiert nach Bennett, 2010:33; Hervorhebung im Original).

Diese Assemblagekausalität versuche ich sowohl anhand menschlicher Akteure als auch anhand spezifischer Dinge, Gegenstände und Situationen aufzuzeigen. Verschiedene menschliche Akteure werden als Zwischenüberschriften auftauchen; die Dinge, Gegenstände und Situationen werden als „Diesheiten“ aufgeführt. Ich werde drei schematische Akteursgruppen dafür herausgreifen: (1) Die Menschen, die ihren Sperrmüll vor die Tür stellen; (2) die Menschen, die spontan und kurzzeitig in das Sperrmüllgefüge hineingezogen werden; (3) das Sperrmüllassemblage als Teil eines größeren Müllsammler_innengefüges, das diverse Menschen aus der Stadt und darüber hinaus anzieht.

\subsection{Den Sperrmüll herausstellen}

\section{Diesheit 1: Kleines blaues Fahrrad}

Während ich eine weitere Straße hinuntergeschoben werde, lehnen ein Kindersitz und ein Buggy an einer Häuserwand. Stopp! Foto! Eine Frau tritt aus dem Haus und stellt ein kleines Fahrrad neben den Buggy: ein kleines blaues Fahrrad. Es hat zwei platte Reifen, ansonsten sieht es gut aus. Zwei Frauen bleiben stehen und schauen sich das Fahrrad an. Nach ein paar Minuten gehen sie ohne das Fahrrad weiter. Ich hingegen frage mich, ob dieses Fahrrad nicht das perfekte Geschenk für das Kind einer Freundin sein könnte. Ich bin zerrissen. Eigentlich will ich doch die affektive Atmosphäre des Sperrmülls untersuchen. Eine weitere Frau kommt aus dem Nachbarhaus. Sie hinterlässt ein Paar ungetragene Snowboardboots. Im Gehen ruft sie mir fragend über die Schulter zu: „Welche Schuhgröße hast Du?“ „44.“ Während sie schon wieder im Haus verschwindet, ruft die Frau noch einmal: „Tja, das ist Pech, dann passen sie Dir nicht! Die waren richtig teuer!“ Tja ... Ich nehme das Fahrrad und bringe es nach Hause. Als ich mit dem Fahrrad unterm Arm weggehe, höre ich, wie die erste Frau zu ihrer Tochter sagt: „Ich wusste gar nicht, dass heute Sperrmüll ist. Hab auf'm Weg von der Arbeit gesehen, wie die Leute ihre Sachen rausstellen. Mal sehen, was wir noch alles im Keller haben.“

In der Situation, die sich um ein kleines blaues Fahrrad herum abspielt, taucht eine Frau auf, die zu einer der drei schematischen Kategorisierungen der menschlichen Akteursgruppen gehört, die in das Sperrmüllassemblage hineingezogen werden und von seinen affektiven Schüben und Zü-

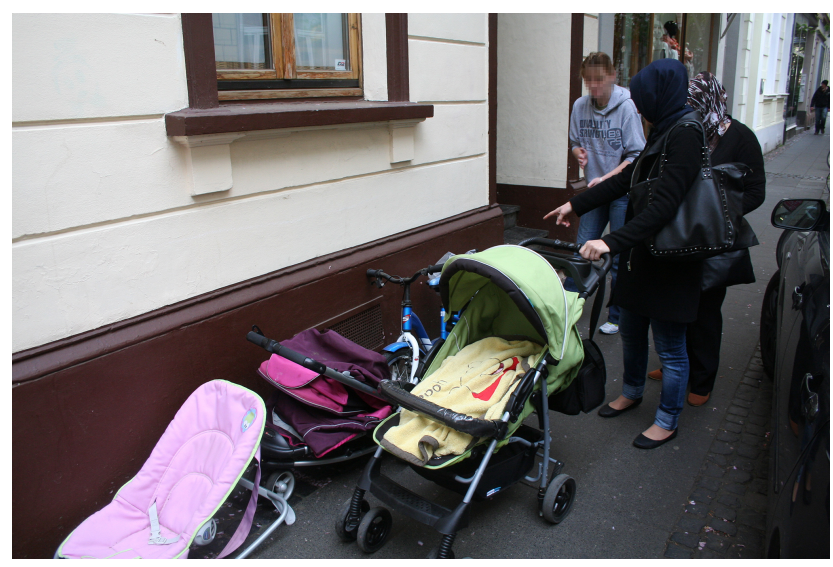

Abb. 1. Kleines blaues Fahrrad.

gen bewegt werden: die Menschen, welche ihren Sperrmüll vor die Tür stellen. Manche davon planen natürlich voraus. Sie tragen sich die Abholdaten in ihre Kalender ein, überlegen, was sie nicht mehr brauchen, um es dann vor die Tür zu stellen, wenn es soweit ist. Andere hingegen werden, z.B. auf dem Weg von der Arbeit nach Hause, vom Sperrmüll überrascht. Die in der Vignette zuerst auftauchende Frau, hat ihre Dinge nicht zum Herausstellen vorbereitet. Sie muss vor Ort und in der Situation überlegen, was sie noch braucht und was nicht. Sie ist in situ/in tempo damit konfrontiert, was Müll (für sie) ist.

Die Frau, die mit Snowboardschuhen die Gehsteigbühne betritt, zeigt uns hingegen, dass manche Menschen um den Wert der wegzuschmeißenden Sachen wissen: den Wert, den sie haben oder den sie für jemanden haben könnten. Auch wenn das bestimmt nicht für alle zutrifft, sind manche Menschen extrem angespannt, wenn sie Dinge wegschmeißen (vgl. Jones und Evans, 2012). Während etwas auf die StraBe herausgestellt wird, finden vielleicht sofort andere Menschen Gefallen an dem, was vorher noch für Müll erachtet wurde (vgl. Abb. 1). Es schien, als ob die Frau in der obigen Diesheit sich schon fast gezwungen fühlte, mich nach meiner Schuhgröße zu fragen. Oftmals haben die Menschen auch nicht mit mir sprechen wollen, als ich sie beim Herausstellen von Dingen angesprochen habe. Die Qualität der Objekte, die man wegschmeißt, kann darüber bestimmen, wie man sich beim Wegschmeißen fühlt. Manche Menschen versehen ihre noch funktionierenden Geräte - die im Übrigen gar nicht auf den Sperrmüll gehören - mit kleinen Zetteln (vgl. Abb. 2) oder kleben die nötigen Schrauben oder Nägel an die zugehörigen Regale oder Schränke. Es lässt sich also bereits erahnen, dass das Herausstellen des Sperrmülls kein trivialer Akt ist.

Ein weiterer Grund, warum sich das Herausstellen von Sperrmüll sonderbar anfühlen kann, könnte der sein, dass sich Menschen nicht genau sicher sind, was sie eigentlich alles vor die Tür stellen dürfen. Wenn man Dinge zum Sperrmüll vor die Tür stellt, bleibt oft eine kleine fragende Sorge 


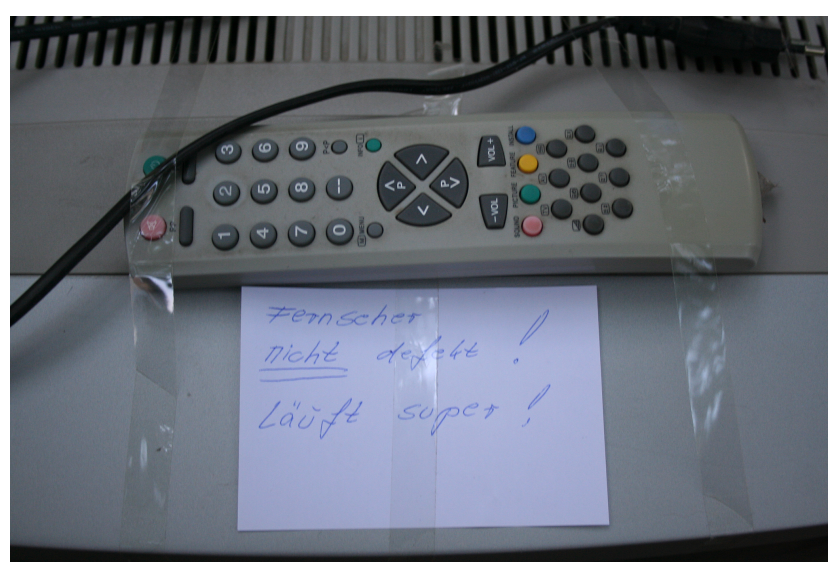

Abb. 2. Fernseher nicht defekt! Läuft super!

zurück, ob das auch Sperrmüll ist, was man da loswerden will (vgl. WDR, 2014). Oftmals gehen die Leute nach einem gewissen Trial-and-Error-Prinzip vor, sagt Birgit Gußmann, die Öffentlichkeitsbeauftrage von bonnorange ${ }^{7}$.

Ein dritter Grund, warum das Herausstellen eine gewisse Unruhe auslösen kann, ist die Möglichkeit, dass unser Sperrmüll genau durchsucht wird. Dieser Aspekt wird auch in einer kleinen Dokumentation des Radiosenders WDR 5 (2014) thematisiert. 2014 haben Starkregenereignisse viele Keller im Nordwesten von Nordrhein-Westfalen überflutet. Viele Gegenstände, die in den Kellern lagerten wurden zerstört. Sie wurden zu Sperrmüll. Allerdings hatten die lokalen Verantwortlichen zu viel mit der Beseitigung anderer Schäden zu tun und so mussten die Leute warten. Während die Menschen auf den Sperrmüllabtransport warteten, wurden sie zusehends wütender auf die „Plünderer“ (,informelle“ Müllsammler_innnen), die durch ihre persönlichen Sachen gingen. Daher bildeten die Betroffenen gemeinsame Nachtwachen, um ihr Eigentum vor der Durchsicht von Fremden zu schützen und die Sammler_innen ,zu vertreiben“. Daniel Miller (2008) beschreibt in seinem Buch The comfort of things eindrücklich, dass wir intensive Beziehungen mit unseren Dingen aufbauen und das diese Beziehungen zwischen uns und den Dingen hin und her fließen und dabei helfen, unser Leben zu stabilisieren. Wegwerfen kann daher eine sehr aufreibende Handlung sein. Allerdings hält uns das intime Verhältnis zu Dingen auch nicht davor zurück, sie in aller Öffentlichkeit wegzuschmeißen. Ganz im Gegenteil, wie Heather Rogers (2005:117) uns daran erinnert, ist Ent-Sorgen auch ein Befreien von den ,shackles of tradition from outworn equipment and ideas. Liberated from repair, reuse and the tending of natural systems, the consumer [is] free to waste".

Die erste Akteursgruppe macht sich auch Gedanken darüber, wie sie die Dinge herausstellt. Während eines Sperr-

\footnotetext{
${ }^{7}$ Die bonnorange AöR ist für den Sperrmüllabtransport in Bonn verantwortlich.
}

müllstreifzugs lief ich an einem Paar vorbei, dass dabei war seinen Sperrmüll aus dem Keller zu holen und auf den Bürgersteig zu stellen. Der Mann legte gerade den Müll ab, als die Frau ihn zurechtwies und sagte: ,Axel, mach es ordentlich!“ Für sie und für andere scheint es wichtig zu sein, den Müll „ordentlich“ zu arrangieren und aufzustapeln (vgl. Abb. 3). Wir sind also beim Wegschmeißen von Sperrmüll dazu gezwungen, auf kleine und unscheinbare Weise mit unserer direkten Umgebung zu interagieren: Schätze die Größe und die Form des Gehsteigs oder der Straße ein, stell den Kram so raus, dass es passt, überdenke die räumlichen Konsequenzen deiner Handlungen.

Interessanterweise wachsen Sperrmüllhaufen oftmals an denselben Stellen. An diesen Stellen lassen sich die kleinen Zusammenspiele zwischen unserer städtischen Umwelt sowie dem menschlichen Gewohnheits-Geist-Körper beobachten. Denn diese Plätze werden nicht nur durch den verfügbaren Raum, sondern auch durch weitere Eigenschaften bestimmt: Oftmals braucht es eine Häuserwand, eine StraBenlaterne oder andere Objekte, gegen die der Sperrmüll gelehnt werden kann. Auch andere Menschen, welche die Herausstellenden beobachten, sind ein wichtiger Einflussfaktor für die Entstehung von Sperrmüllhaufen; ebenso die Distanz zum Eingang des eigenen Zuhauses. Wer sich z.B. Abb. 4 anschaut, bekommt einen Eindruck von einem Ereignis des Herausstellens: Ein Mann stellt seinen Sperrmüll vor sein Haus. Während er dies tut, durchstöbern schon andere seine Gegenstände (1). Während der „Wegwerfende“ noch weitere Dinge holt, die er gerne loswerden möchte, bedient sich bereits ein „Sammler“ an den Ressourcen (2). Als der Wegwerfer zurückkehrt scheint er zunächst etwas darüber verunsichert, wo sein Eigentum hin ist (3). Er kehrt abermals in das Haus zurück, um weitere unnötig gewordene Dinge zu holen. In der Zwischenzeit hat der Rest seiner Sachen weitere Interessierte angezogen. Diese nehmen fast alles mit, was noch übrig ist (4 und 5). Der Wegwerfer kehrt abermals zurück und sieht sich nur mit einem einzigen Gegenstand konfrontiert: einem Drucker. Vielleicht ist es sein Drucker, vielleicht auch nicht. Für ein paar Momente steht er still da und blickt auf die fast leere Stelle vor seiner Eingangstür (6). Dann entschließt er sich den Drucker aufzuheben (7) und an den benachbarten Haufen an der Straßenecke zu stellen (8), wo der Drucker bis zu seinem Abtransport auch verbleibt.

Zusammenfassend lässt sich also sagen, dass wir und unser Müll gesehen werden können, wenn wir uns sperriger Gegenstände entledigen. Und das heißt somit auch, dass wir uns beim Herausstellen des Sperrmülls, bewusst oder unbewusst, mit den materiellen Eigenschaften des Mülls auseinandersetzen müssen, ebenso wie mit den Wertvorstellungen und Bedürfnissen von uns selbst sowie von anderen Menschen. Beim Wegwerfen wird unser Standpunkt zur Konsumgesellschaft erfragt (vgl. Gregson et al., 2007). Viel wichtiger ist aber noch, dass diese einst privaten Objekte in eine Unzahl von kleinen Zahnrädern verwandelt werden, die sich gegen- 

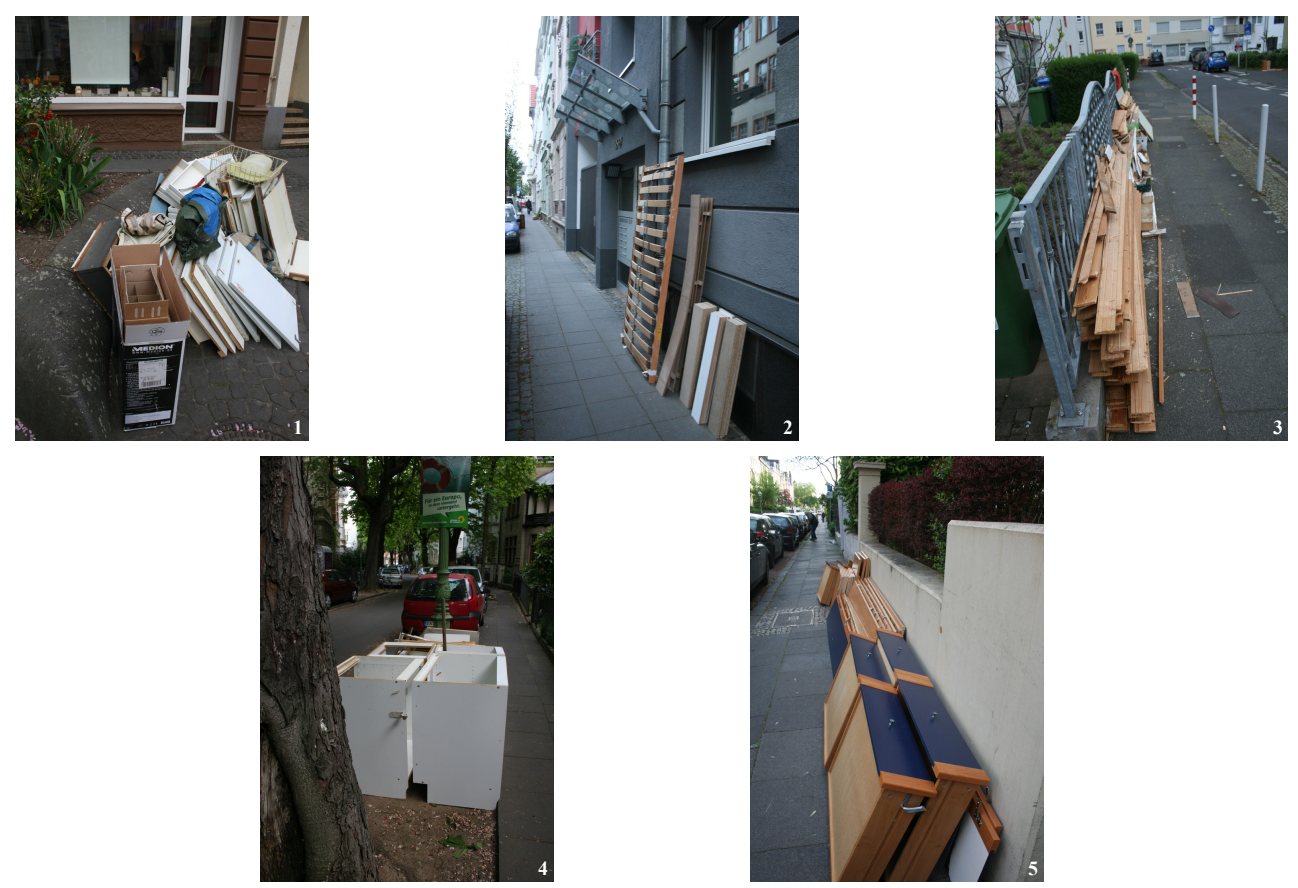

Abb. 3. „Mach es ordentlich!“

seitig als eine quartiergroße Sperrmüllmaschine in Gang setzen.

\subsection{Den Müll mitnehmen oder die Folgen der Wunsch-Maschine}

Um das kleine blaue Fahrrad herum lässt sich noch eine weitere schematische Akteursgruppe erkennen: die Menschen, die kurzerhand und nur kurzzeitig aktive Intervenienten werden. Menschen, die auf dem Nach-Hause-Weg oder auf dem Weg zum Supermarkt sind, können unweigerlich in das Sperrmüllgefüge hineingezogen werden. Sie sehen etwas, fühlen sich erinnert oder berührt, Ideen und Emotionen entstehen. So geht es z.B. zwei Mädchen, die gerade aus dem Supermarkt kommen. Die Arme voller Einkäufe, wollen sie eigentlich nach Hause gehen. Weil der Sperrmüll in ihrer Heimatstadt anders organisiert ist, werden sie vom gemeinsamen Sperrmülltermin in der Bonner Altstadt überrascht. Sie laufen euphorisch entdeckend durch die Straßen und beladen ihre Arme mit weiteren Dingen. Als ich sie zehn Minuten später an einer anderen Straßenecke wieder treffe, haben sie bereits einen Einkaufswagen aufgetrieben und noch mehr Sachen eingeladen (vgl. Abb. ; Foto 5). Die sammelnden Menschen, mit denen ich in den Sperrmülltagen gesprochen habe, sind oft überrascht, was andere Menschen rausstellen oder auch, was man glaubt zu brauchen. Müll fasziniert und bewegt und so hallen in den Straßen die Echos von Deleuze und Guattaris (1977:368) Wunsch-Maschine, in der das Subjekt von einem Assemblage verdrängt wird, in dem Verbindungen geschaffen werden: ,[S]o wird die unmittel- bare Verbindung von Maschine und Wunsch erkennbar, die Maschine geht ins Innere des Wunsches ein, die Maschine wird Wunschmaschine und der Wunsch Maschine. Nicht ist der Wunsch im Subjekt, vielmehr die Maschine im Wunsch und auf der anderen Seite, neben der Maschine und in ihrem Umkreis, ist das residuale Subjekt, Parasit der Maschine und Anhängsel des Wunsches des maschinenartigen Säugetiers.“ Die Wunsch-Machine ist ein zentrales Konzept von Deleuze und Guattari, um die Freud'sche Vorstellung vom Unbewussten und der damit einhergehenden politischen und ökonomischen Vorstellung vom Menschen zu zerstören. Kurzgefasst verstehen Deleuze und Guattari unter dem Unbewussten eine produktive „Maschine“, die konstant Wünsche produziert. Das Unterbewusstsein als Wunsch-Maschine ist nicht mehr vom Mangel getrieben, nicht mehr etwas, was die Beziehung zwischen Kindern und Eltern repräsentiert, sondern es ist die Basis für die soziale (Re)Produktion (Buchanan, 2008:39) Wunsch verstanden als Motor, als Produktion, das Leben als Produktion (Deleuze und Guattari, 1977). Das Leben wird als ein Mechanismus verstanden, in dem alles verbunden ist und der in andere Maschinen und Gefüge eingestöpselt ist. Ein Subjekt ist dementsprechend auch nicht zu einem einmaligen Zeitpunkt entstanden und agiert fortan aus einem endlichen Produktionsprozess heraus, sondern es wird kontinuierlich produziert und neu zusammengesetzt; es wird konstituiert, within the given or experiences but also imagines or projects to the not-yet given future" (Colebrook, 2002:81). Erfahrungen und Wahrnehmungen produzieren Bilder oder „Investitionen“. 
The child's mouth, for example, that has experienced pleasure at the breast comes to desire or anticipate the breast. (...) but for Deleuze desire is more than the actual. In the case of the child, the mouth's past pleasure produces an idea or image of further pleasure, and this creates an ,investment“. The breast becomes more than what it actually is (a body part) and takes on an added virtual dimension - the breast of fantasy, pleasure and desire (ebd.:82).

Der Wunsch entstammt immer den präpersonalen, affektiven Erfahrungen, die erst hinterher in Institutionen (MutterKind-Beziehung, der Familie, Kultur) eingehegt werden (ebd.). All das bedeutet, dass die affektiven materiellen Begegnungen, die wir machen, Wunschgefüge befeuern. Es gibt nie nur den einen Wunsch; wir stecken immer in Wunschgefügen. Wir wünschen uns nie irgendetwas; wir wünschen uns immer nur Wunschgefüge (Deleuze und Guattari, 1992). Wir sind eingestöpselt in einen allgegenwärtigen Fluss des Lebens (des Wunsches), welche Bedürfnisse und Sehnsüchte produziert: beides sowohl aktualisierte als auch virtuelle.

Das Laufen durch das Sperrmüllassemblage zeigt uns, dass alle Aspekte - Gedanken, körperliche Funktionen, Erinnerungen, die Luft, die wir atmen, die Kälte der Nacht, das Wetter des Tages, die Gegenstände, die Dinge und Menschen, die wir sehen usw. - zusammen gehören und uns auf molekularer Ebene verändern und so permanent Wünsche produzieren. Daher kommt es auf „Intensitätsstreifen, Potentiale, Schwellen und Gradienten“" an (Deleuze und Guattari, 1977:27). Wenn einmal eine affektive Situation einen Schwellenwert überschreitet, wird etwas Neues produziert: Auch wenn es nur der Wunsch ist, ein kleines blaues Fahr$\mathrm{rad} \mathrm{zu}$ verschenken. Wobei der Wunsch ein kleines blaues Fahrrad zu besitzen bereits wieder eine Territorialisierung des Wunsches bedeutet (Buchanan, 2008:119-120). Ein weiter tragendes Beispiel ist mein Freund Alexander, welcher eines Sperrmüllabends über eine hölzerne Palette stolperte. Er erzählte mir, wie er ohne die Assoziation begründen zu können, an die Bilder eines Konzentrationslagers erinnert wurde und an die eintätowierten Nummern der Häftlinge. Seinen ersten Eindruck verarbeitet er in einem kleinen persönlichen Kunstwerk, was heute in seinem Wohnzimmer (voll mit zahlreichen Sperrmüllfunden) hängt (vgl. Abb. 5). Die Palette hat er mit einem Stencil des jüdischen Künstlers Charlie Chaplin und einer fiktiven KZ-Nummer versehen.

Nicht immer kann das Laufen durch das Sperrmüllassemblage so direkt theoretisiert werden. Es überrascht durch seine Rohheit von Objekten, seinen Bauschutt, seine kaputten Bretter, seine alten Türen und Fenster, die alten Farbeimer, kaputte elektronische Geräte oder Toilettensitze. All diese Dinge scheinen am Ende ihres Lebenszyklus zu sein. Sie zeugen von Renovierungs- und Restaurierungsarbeiten oder, wie Stephan Graham und Nigel Thrift (2007:17) es nennen, von der ,invisible work of maintenance and repair“", welche die Ordnung unseres täglichen Lebens aufrechterhält. Maintenance and repair bedeutet, dass neben leicht verwertbaren Stoffen auch Dinge weggeschmissen werden müssen, die nicht einfach ,verschwinden“ können, wie z.B. Farb- oder Lackreste oder gewisses Plastik (vgl. Thompson, 2013). Die Glattheit unseres Lebens ist also direkt verbunden mit der Rohheit und der Entsorgung von Dingen. Denn Müll verschwindet nicht; er wechselt nur die Form und entschwindet unseren Augen.

Ein weiteres Beispiel, wie der Sperrmüll Dinge in Gang bringen kann, ist das Beispiel der Aktion Kurze an die Wand des Bonner Kulturvereins Rhizom. Diese lädt dazu ein, sich an lauen Sperrmüllnächten Sitzgelegenheiten vom Sperrmüll zu besorgen und diese an eine vorher ausgemachte Stelle zu bringen. Das Rhizom bringt eine Leinwand und einen Beamer mit und projiziert, meist zu Livemusik, Kurzfilme an Gebäudewände (vgl. Abb. 6). Der Sperrmüll gibt hier dem öffentlichen Raum eine utopische Qualität (vgl. Belina, 2006:201-204). Menschen bauen für den kurzen Moment ein Freiluftkino, das jedes Mal anders aussieht. So füllt sich ein Schulhof plötzlich mit Sofas, Stühlen, Kisten und Truhen und Menschen treffen an einem Ort aufeinander, den sie sonst täglich passieren. Anwohnende müssen sich jedoch auch mit der ungewöhnlichen Nutzung auseinandersetzen. Neben einer kontroversen Debatte um die Nutzung des öffentlichen Raums (vgl. Smith und Low, 2006) kann Sperrmüll auch weitere (alltags-)politische Debatten auslösen. Die zweite Diesheit im nächsten Kapitel soll dabei helfen, diese Aussage besser verständlich zu machen.

\subsection{Die professionellen Sperrmüllsammler_innen}

Der Sperrmüll produziert als dritte Akteursgruppe Sperrmüllsammler_innen, welche die Straßen an Sperrmülltagen nach Gegenständen durchsuchen, die wieder zu verkaufen sind. Im Durchsuchen von Sperrmüllhaufen sind diese Menschen meist geübt, weniger zaghaft und zielgerichtet. Bei vielen studentischen Altstadtbewohner_innen kann, aufgrund der Dinge, die sie sich anschauen und sammeln, der Eindruck entstehen, dass sie die Gegenstände vom Sperrmüll nicht nur einstecken, weil sie kostenlos sind, sondern auch weil sie zu einem Umwelt-Retro-Lifestyle passen. Wohingegen die professionellen Sperrmüllsammler_innen Gegenstände auch nach ihrem ökonomischen Wert hin mitnehmen. Sie lassen auch Gegenstände in ihre Kastenwägen verschwinden, die aufgrund ihrer Beschaffenheit, z.B. weil sie aus (Edel)-Metall sind, einen Materialwert, aber sonst keinen Nutzen haben (siehe unten).

Diese Kastenwägen sind während der Sperrmüllzeiten in der Bonner Altstadt allgegenwärtig. Hört man hier (vgl. Track 1 (doi:10.5446/20233)) in die Straßen der Altstadt, dann lässt sich erahnen, wie die knatternden Lieferwägen Teile der Tonspur des Sperrmülls bilden und sich tief ins auditive Gedächtnis eingraben. Es sind die Geräusche von blubbernden Dieselmaschinen im Leerlauf, die Geräusche 

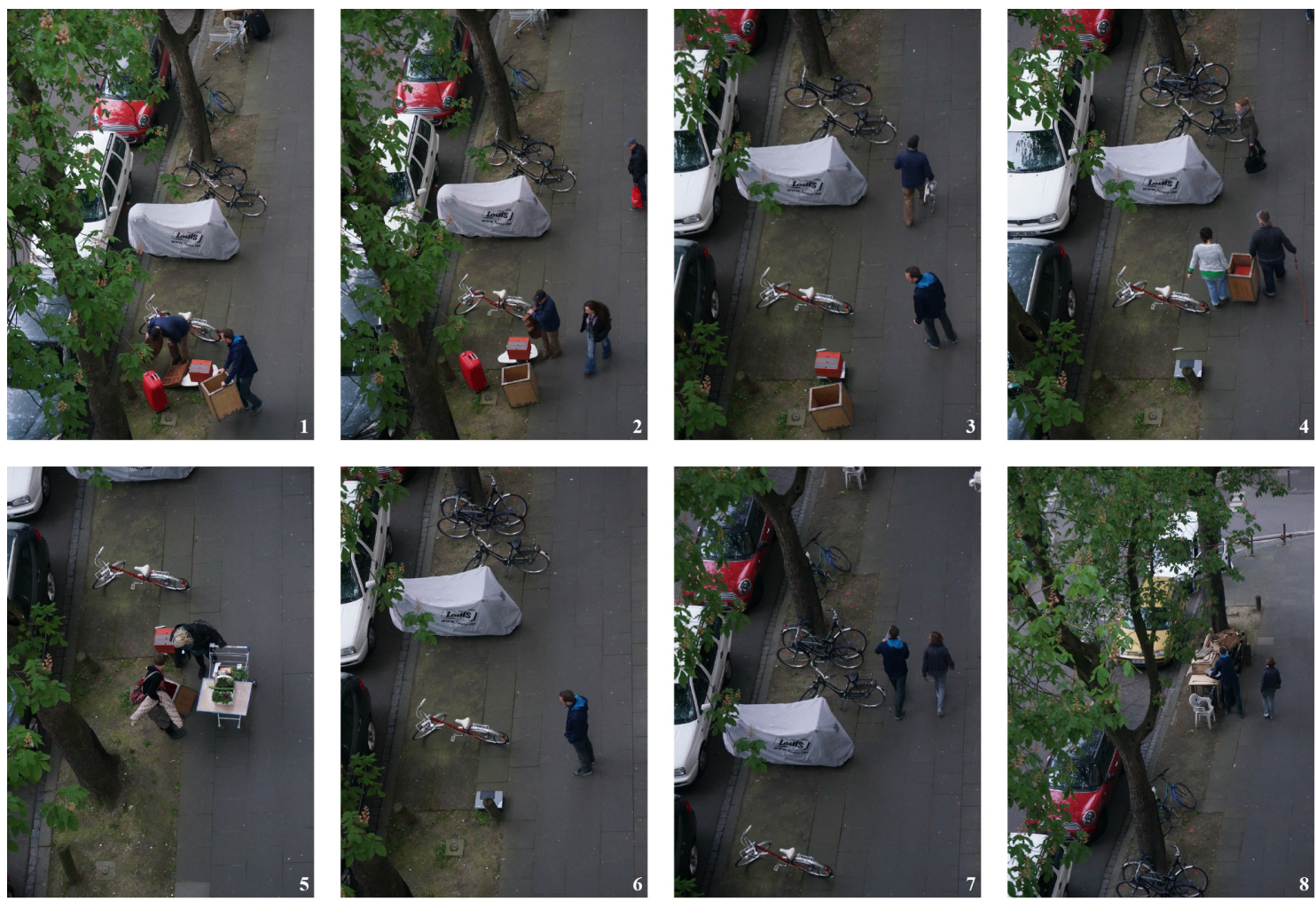

Abb. 4. Den Sperrmüll herausstellen.

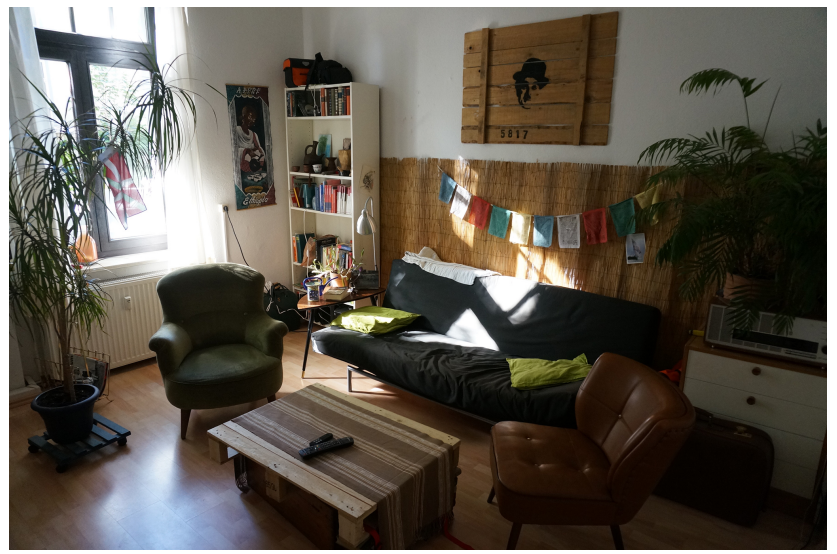

Abb. 5. Alexanders Wohnzimmer.

von sich öffnenden und schließenden Türen, es ist das $\mathrm{Hu}-$ pen, das Aufheulen von Motoren, das nachklingt, wenn man schon lange wieder zu Hause ist. In den Kastenwägen wird der Platz oft geschickt bis unter die Decke und auf dem Dach ausgenutzt, um die sperrigen Fundstücke unterzubringen. Die meisten dieser Kastenwägen haben osteuropäische Nummernschilder (vgl. Abb. 7).

Die Aktivitäten dieser sogenannten ,informellen“ Müllund Wertstoffsammler_innen sind, bezogen auf den Spermüll in Deutschland, kaum erforscht (vgl. Obersteiner et al.,

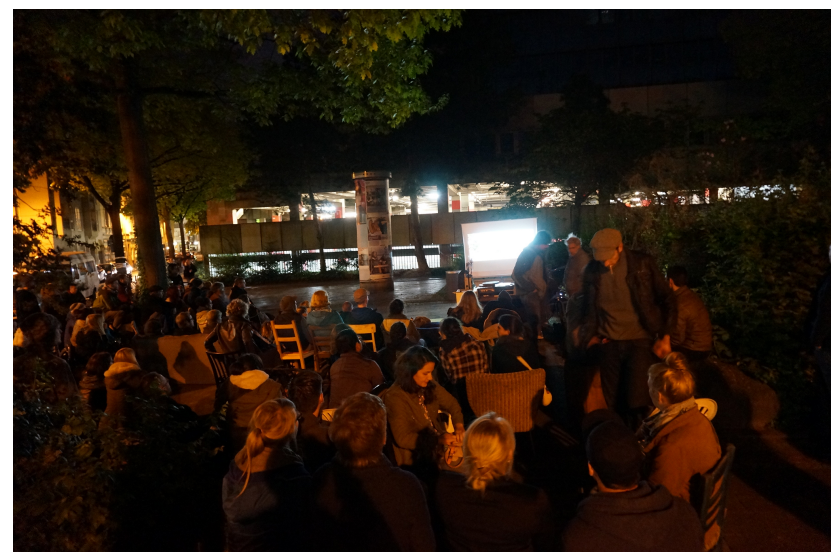

Abb. 6. Kurze an die Wand.

2010). Dementsprechend gibt es wenig Literatur - nicht zuletzt auch deshalb, weil es ohne Übersetzer_in nicht einfach ist, mit den Sammler_innen in Kontakt zu kommen. Das einzige Forschungsprojekt, das ich finden konnte, welches sich aus einer sozialwissenschaftlichen Perspektive mit dem europaweiten ,informellen“ Müllsammeln und -abtransport beschäftigt hat, heißt TransWaste und lief von 2009-2012 (ebd.). Sperrmüll war nur ein Teilbereich des Projektes. Das Projekt untersuchte u.a. die ,informelle“ Sperrmüllsammlung in Deutschland und Österreich. Es hatte die Beantwor- 


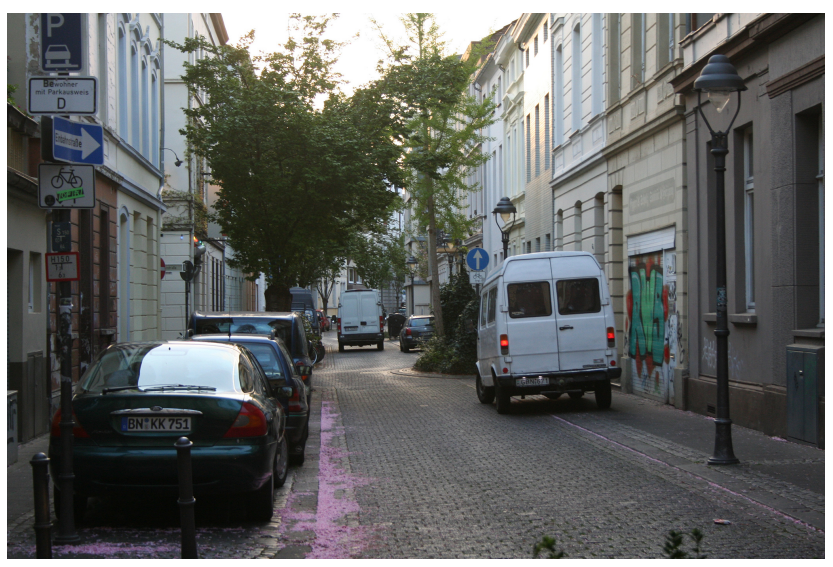

Abb. 7. Kastenwägen durchkreuzen die Altstadt.

tung zweier Fragen zum Ziel: „(1) to find out exactly what informal activities are carried out and (2) their relevance in comparison to formal waste collection activities" (Obersteiner et al., 2010). Laut TransWaste kommen die meisten Menschen, die in Deutschland den Sperrmüll durchsuchen, aus Rumänien, Ungarn, Polen, der Tschechischen Republik und Litauen (ebd.). Die Daten über Österreich lassen den Schluss zu, dass die Müllsammler_innen aus Osteuropa meist sehr arm sind und versuchen, durch das Sperrmüllsammeln ,über die Runden zu kommen“. Ein Viertel der für Österreich Interviewten gaben an, dass Sperrmüllsammeln die einzige Möglichkeit sei, Geld zu verdienen (ebd.). Monika Żurańska-Skalny (2010) berichtet darüber, dass $90 \%$ aller befragten Sperrmüllsammler_innen angeben, dass durch das Sammeln von Sperrmüll ihre Gesundheit negativ beeinflusst wird. Die bevorzugten Gegenstände sind Möbel (z.B. Regale, Stühle oder Sofas), Haushaltsgegenstände, Metalle und elektronische Geräte (z.B. Kühlschränke, Monitore oder Computer) (Obersteiner, 2010). Die Gegenstände werden in die jeweiligen Heimatländer gebracht und dort auf Märkten verkauft (Obersteiner et al., 2010). TransWaste schätzt, dass die abtransportierte Menge an Abfall für Österreich ca. $15 \%$ des offiziell eingesammelten Mülls beträgt (ebd.). Laut der WienerZeitung (2012), welches ein Interview mit dem Forschungsprojekt geführt hat, ist die Ökobilanz durch Wiederverwendung durch die Sammler_innen wesentlich besser als durch Materialrecycling. Durch die erneute Nutzung von Plastikstühlen, Kleidung oder Autoreifen werden 19 bis $48 \%$ der Treibhausgasemissionen eingespart. Eine Ausnahme bilden alte Elektrogeräte: Deren Weiterbenutzung bedarf wesentlich mehr Energie als die Nutzung von neuen Geräten (ebd.). An der Frage von Wiederverwertbarem setzt die folgende Diesheit an.

\section{Diesheit 2: Das Öffnen eines Fernsehers}

Ein vom Leben gezeichneter Mann sitzt auf einer Bank und schraubt unwirsch einen Flachbildschirm auf. Nähertreten, zuschauen und fragen: „Kann ich ein Foto von Ihrem Fund machen?“ Irritation. „Warum?“. „,̈h, weil ich etwas über den Sperrmüll schreibe.“ Leuchtende Augen. Begeisterung. Zustimmung. ,Das ist eine gute Idee! Die Menschen wissen gar nicht, was sie alles wegschmeißen! Daher schraube ich auch dieses Monster hier auf.“ Der Schraubenzieher rutscht ab und fährt ihm in die Hand. „Alle drei bis vier Monate bringe ich das Metall zur Sammelstelle und kriege so 300, $400 €$ dafür. Hab' sogar schon einen Keller von 'nem Kumpel als Lager gemietet. Alles legal beim Finanzamt angemeldet. Aber diese ganzen Ausländer, die hier herumlaufen. Die nehmen die ganzen guten Sachen und bringen die zurück in ihre Länder. Ich habe mich eben beinahe mit zwei von denen geprügelt. Die wollten mir etwas aus meinem Handkarren klauen. Als ich mich gewehrt habe, haben die mich einen Nazi genannt. Das war zu viel! Hätte ich nicht schon 'ne Vorstrafe wegen einer ähnlichen Sache, hätte ich die zu Brei geschlagen!“ Mittlerweile liegt das Innenliegende des Bildschirms offen. „Ah, das hat sich doch gelohnt: Dafür kriege ich bestimmt fünf Euro.“

Den Sperrmüll nach wertvollen Materialien zu durchsuchen scheint also keine einfache Aufgabe zu sein. Der Mann, der den Bildschirm aufschraubt, muss hart für sein Geld in der Kälte arbeiten. Seine Hände sind voller Schwielen und die Konkurrenz bei der Wertstoffsammlung scheint konfliktbereit zu sein. Der Sperrmüll zieht Menschen aus der Stadt und ihrem Umland sowie aus anderen Ländern Europas an, die dann „unseren“ Abfall sammeln. Unsere (westeuropäischen) gebrauchten Gegenstände haben einen hohen Wert für diese Menschen aus Osteuropa (Obersteiner, 2010). So macht der Sperrmüll den ökonomischen Gradienten innerhalb der Europäischen Union und natürlich auch innerhalb der Stadt an manchen Tagen mitten in der Altstadt unmittelbar erfahrbar. Er produziert eine Überlappung von unterschiedlichen finanziellen Räumen, die an anderen Tagen weit entfernt voneinander scheinen. Im Sperrmüll zeigen sich so globale Ungleichheiten gleichsam vor der Haustür und Fragen von (Un-)Gerechtigkeit werden verhandelt. Menschen besitzen nicht nur, sondern werfen sogar das weg, womit andere Menschen ihren Lebensunterhalt erst sichern. Jean-Luc Nancy (2011:43) schreibt in einem kleinen Essay über Gerechtigkeit, dass ein altbekanntes Verständnis von Gerechtigkeit besagt: „to render to each his due“ oder „to give to everyone what he is owed“. Diese knappe Definition von Gerechtigkeit hat zwei inhärente Prinzipien. Das erste ist „Gleichheit": Jede_r ist gleich viel wert. Das andere Prinzip ist „Differenz“ oder „Singularität“: Was einer Person zusteht, steht nicht notwendigerweise auch einer anderen Person zu. Daraus folgt, so Nancy, das Gerechtigkeit immer nur relational gedacht werden kann. Was für mich gerecht oder ungerecht ist, kann nur anhand dessen bestimmt werden, was gerecht oder ungerecht für jemand anderen ist (ebd.). Fragen nach der Legitimität von Besitz werden in einer Situation deutlich, in der mehrere Kastenwägen plötzlich die engen Straßen der Altstadt blockieren. Ein älterer Passant bemerkt laut- 


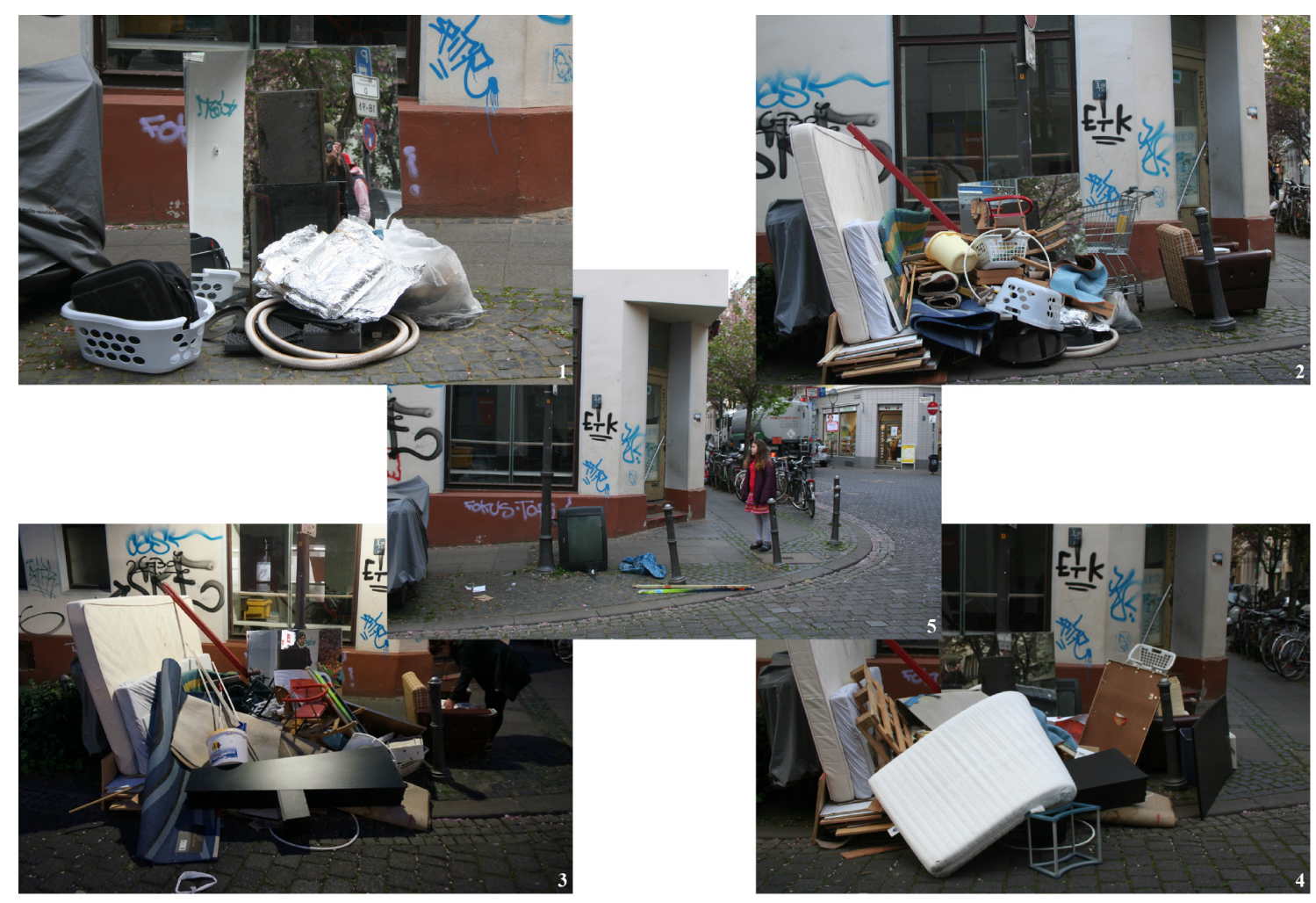

Abb. 8. Werden eines Sperrmüllhaufens.

stark: „Wie die Geier!“ (vgl. Track 2 (doi:10.5446/20233)). Er vertritt offenbar den Standpunkt, dass gewisse Menschen, die in der Altstadt den Sperrmüll durchsuchen, diesen nicht verdient haben - ähnlich wie der Mann, der den Fernseher aufschraubte und sich ungerecht behandelt fühlt, wenn „Ausländer" den Bonner Sperrmüll wegnehmen. Nancys kleines Essay über Gerechtigkeit macht deutlich, dass ich erst in Bezug zu anderen Menschen Un-/Gerechtigkeit empfinden oder ausdrücken kann. Vielen Menschen geht es natürlich auch ganz anders. Die zerbeulten und lauten Kastenwägen bringen viele Altstadtbewohner_innen unweigerlich da$\mathrm{zu}$, ihren Wohlstand in Relation zu sehen und Ungleichheit aushalten zu müssen. Innereuropäischer Transit. Innereuropäisches Wohlstandsgefälle. Ob er nun wütend wird, was andere Leute sich herausnehmen oder ob ich traurig darüber bin, was mir zusteht und anderen nicht, das Herausstellen von Gegenständen initiiert Interaktionen zwischen den vorgestellten Gruppen. Manche Menschen aus Osteuropa treten auch in direkten Kontakt mit den Anwohner_innen und fragen nach spezifischen Gegenständen. Doch auch die verschiedenen sammelnden Personen treten in Kontakt und stehen nicht selten in Konkurrenz zueinander. Die Kastenwägen (weniger die Menschen in ihnen, mit denen selten direkter Kontakt entsteht) werden von manchen Sammler_innen, die sich erst Transportmittel organisieren müssen, als ,stressig“ empfunden. Außerdem wird so mancher Schrank durch bereits zügig entfernte Metallscharniere, Griffe etc. wertlos für andere Sammler_innen oder Liebhaber_innen alter Möbel. Auch wenn diesen Sammler_innen wohl bewusst ist, dass ökonomische Zwänge moralisch über der eigenen Freude am Sammeln stehen, wünschen sich manche spontane Schatzsucher_innen in Ruhe suchen zu können. Mein bereits erwähnter Freund Alexander bemerkte einmal, frustriert über alte zerstörte Möbel, dass er sich wünsche, es gäbe eine Möglichkeit, die Situation während des Sperrmülls einzufrieren, sodass man in Ruhe nach Schätzen suchen kann und erst danach alles durchwühlt, zerstört und abtransportiert wird. Selbstverständlich werden „Schätze“ dabei für alle Akteur_innen sehr unterschiedlich bewertet und allen käme vermutlich manchmal ein Einfrieren gelegen. Doch werden sie eben durch affektive Züge und Schübe des Sperrmülls erst bewegt. Sie alle verändern die Situation unabhängig voneinander in unvorhergesehener Weise. Die Aktion einer Person ist direkt mit der Handlung einer anderen Person verbunden, ganz zu schweigen vom Eigenleben der Gegenstände, die sich als selbstständige Akteure einfügen. Alle diese separaten aber kollektiven Handlungen verändern die Sperrmüllhaufen im Laufe der Nacht fast, wie es scheint, von einer unsichtbaren Hand angeleitet (vgl. Abb. 8). Nicht selten lassen sie Bürgersteige überlaufen, sodass der Verkehr sich staut, Bürgersteige unpassierbar werden und die Müllmänner in mühevoller Kleinarbeit die Straßen säubern müssen. 


\section{Fazit: Sperrmüll ökologisch denken}

The most extraordinary things are also the most everyday; the strangest things are often the most trivial, and the current notion of the "mythical" is an illusory reflection of this fact. (Lefebvre, 1991:13-4)

Es ist genau diese Faszination für die außergewöhnlichen alltäglichen Ereignisse, die Henri Lefebvre beschreibt, die mich dazu verleitet hat, mich mit dem Sperrmüll zu beschäftigen. Die Tage und Nächte, in denen der Sperrmüll in der Altstadt auf die Straße gestellt wird, produzieren Möglichkeiten, potentielle Projekte, aber auch intime Fragen. Gleichwohl ist es erst eine nicht-repräsentationale Denkweise, welche die Vielheiten und die Verbindungen in den Blick nehmen will, die mir hilft, mich dem Sperrmüll als banales Ereignis der Daseinsvorsorge akademisch nähern zu können. Für meine Auseinandersetzung mit dem Sperrmüll stütze ich mich auf drei methodologische Pfeiler. Erstens ermöglicht mir die Forderung, dass nicht-repräsentationales Vorgehen auch immer ein Experimentieren sein muss, ein neues Verständnis von Theorie und Empirie, in dem es nicht darum geht, die Welt so treu wie möglich abzubilden, sondern einen neuen Zugang zur Welt zu kreieren. Dabei spielt, zweitens, der forschende Körper eine wichtige Rolle. Es geht darum, sich eine Art materielle Denkweise anzueignen, die es erlaubt, sich durch das eigene Werden mit den zu untersuchenden Materialitäten in Verbindung zu setzen. Das Gehen, genauer das psychogeographische Umherschweifen, ist dabei eine hilfreiche Stütze. Gehen ist eine Begegnung mit dem, was uns umgibt und es lässt sich erfahren, dass sich unsere Subjektivitäten durch konstante affektive Zusammentreffen mit uns und unserer Umgebung entfalten (Wylie, 2002, 2005). Schließlich ist, drittens, das Schreiben, als ein aktiver und kreativer Prozess, für ein nicht-repräsentationales Vorgehen von elementarer Bedeutung, da es neue Verbindungen schafft und uns das Da-zwischen näher bringt. Deleuze (1993:57-58) formuliert dies wie folgt: „In gewisser Weise handelt es sich immer darum zu stottern. Nicht im Sprechen zu stottern, sondern in der Sprache selbst (...) [Es geht] darum, Fremder in der eigenen Sprache zu sein." Erst durch das Fremdwerden - Fremdwerden sowohl in der Annäherung an den Untersuchungsgegenstand als auch das Fremdwerden in seiner Präsentation - zeigen sich weitere Verbindungen. In Teilen mag das an die Geertz'sche (1983) „Dichte Beschreibung" erinnern. Allerdings geht es beim materiellen Denken eher um den Versuch, sich vom Materiellen affizieren und leiten zu lassen. Denn Handlungen entstehen im Wechselspiel der sich verbindenden Körper (Anderson und Harrison, 2010:4-5). Es geht nicht darum, sich auf soziale Handlungen zu konzentrieren, diese so ganzheitlich wie möglich wiederzugeben und deren Sinngebung herauszuarbeiten (ebd.), sondern eine ,speculative onto-story“ (Bennett, 2010:3-4) zu schreiben, in denen einzelne Verbindungen lebendig hervor- dringen (Vannini, 2015) und wie scheinbar zufällig Brücken $\mathrm{zu}$ anderen Bereichen schlagen. Bei diesem Verbindungsgenerieren, das keinen Unterschied zwischen Aktualisiertem (aktual) und Nichtaktualisiertem (virtuell) macht - unterstützt mich Deleuze und Guattaris Assemblagekonzept. Es verhilft mir dazu, den Sperrmüll als die quartiergroße Maschine zu sehen, die Menschen und Materialitäten in einer einzigen Assemblagekausalität verschmelzen lässt. Menschen, die Sperrmüll herausstellen, produzieren viele kleine Rädchen und setzen diese in Gang. Manche dieser Menschen setzen sich während des Sperrmüllherausstellens mit ihrem Müll auseinander und dadurch mit ihren eigenen und fremden Bedürfnissen sowie im Kleinen mit den räumlichen Konsequenzen ihrer Handlungen. Weiterhin zeigt der Sperrmüll, wie unsere Aktivitäten immer in Gefügen eingebettet sind, die Bedürfnisse und Sehnsüchte produzieren und über das Momentane hinausreichen. Sobald wir eine (gewisse oder ungewisse) Schwelle überschreiten, können wir in neue Beziehungen eintreten, die wiederum neue Geschwindigkeit und Langsamkeit hervorbringt. Auch wenn dies vielleicht bedeutet, erst einmal einen Einfall für ein Kunstwerk zu haben. Außerdem erhält der öffentliche Raum durch den Sperrmüll eine neue qualitative Dimension. Eine Art urban common entsteht. Wir können uns alter Dinge bedienen und neue Dinge entstehen lassen, z.B. ein Freiluftkino. Zudem ist es möglich, mit Gegenständen außerhalb unserer gewohnten Umgangsformen in Kontakt zu kommen. Wir können die Dinge aufheben, aufsammeln, mit nach Hause nehmen, sie an anderer Stelle hinschmeißen oder sogar kaputtmachen. Wir können neue affektive Erfahrungen mit den Dingen des Alltags sammeln und somit einen anderen Zugang zu unserer Umwelt erhalten. Daneben macht das Sperrmüllassemblage uns das innerstädtische und innereuropäische Wohlstandsgefälle augenscheinlich. Ein ganzes System hat sich etabliert, in dem Menschen bspw. von Polen nach Deutschland fahren, um dort das zum Leben Notwendige einzusammeln, was andere Menschen wegschmeißen. Durch das Sperrmüllassemblage werden wir mit anderen, normalerweise weit entfernt lebenden Menschen und ihren Schwierigkeiten verbunden, wodurch wir mit der Frage nach (Un-)Gerechtigkeit konfrontiert werden können. Das Sperrmüllassemblage ist somit eine Art „affective habit ecology“ (AHE) (Dewsbury, 2012), in der wir erleben können, dass unsere Handlungen vielmehr im materiellen Setting der Stadt eingebettet sind, als wir das üblicherweise wahrnehmen. Sperrmüll als AHE zu theoretisieren bedeutet, dass er als ökologische Umgebung gesehen werden kann, der sich der Mensch subtil und fast unmerklich anpasst. Sperrmüll ist aber auch eine Umwelt, in welcher neue Gedanken und Handlungen entstehen können (ebd.). Der Sperrmüll als AHE ist eine Chance, einem der ethischen Grundsätze von neo-materialistischen Ansätzen zu folgen. Nämlich die jeweiligen Gefüge, deren Teil man ist und die gewisse Handlungen vereinfachen und andere erschweren, zu hinterfragen und sich ggf. in andere Gefüge hinein zu begeben (vgl. ebd.; Bennett, 2010:37). 
Diese, zugebenermaßen vielleicht etwas fremd anmutende, Herangehensweise bedeutet allerdings nicht, dass die Ergebnisse einfach produziert werden können. Das Scheitern ist ein ständiger Begleiter (Dewsbury, 2009). Warnungen wie die von Dewsbury (2011a:149, meine Hervorhebung) hallen in den unterschiedlichen Situationen im meinem Kopf wider:

[W] hile the assemblage concept configures a dramatically different way of conceiving the world (...), its arrival does prefigure a dangerous trend to present an ever-growing undifferentiated magma of assemblages (becoming everything, amounting to nothing) resulting in ,piecemeal appropriation“ and „middle-range theorizing“".

Dementsprechend bin ich konstant besorgt, dass mein Vorgehen entweder nichts ergibt oder ein Stückwerk bleibt. Gleichermaßen ist es eine Herausforderung, einen passenden Schreibstil zu finden - ein Schreibstil, der sowohl abstrakt genug ist, und somit einen Einblick in die Potentialitäten/Virtualitäten des Sperrmülls gibt, als auch einer allzu schnellen Kategorisierung entflieht. Das verlangt eine gewisse literarische Fingerfertigkeit. Obwohl eine Pluralisierung der wissenschaftlichen Schreibstile durch die Neuen Kulturgeographie auch in den deutschsprachigen Humangeographien eingesetzt hat (Lossau, 2007:68), lernt man in (deutschsprachigen) Universitäten bisher selten diese ,gewisse essayistische Leichtigkeit" (ebd.), die auch für eine nicht-repräsentationale Denkweise gebraucht werden kann.

Aber genau diese Schwierigkeiten sind nicht bloße Komplikationen, sondern sie machen NRT im Kern aus, eben gerade weil eine nicht-repräsentationale Denkweise nicht zum Ziel hat, die inhärenten Spannungen in formgenaue Worte zu separieren - sie einzufangen, sie zu messen oder sie zu erklären - sondern weil sie zum Ziel hat, unsere Sensibilität gegenüber der Übermäßigkeit des Lebens zu erhöhen (Roberts, 2012:2517). Dementsprechend ist das Ringen mit der (Un-)Möglichkeit, dieser posthumanen Dimension gerecht zu werden, genau das Ziel von NRT. Non-representational thinking fordert uns dazu auf, eine Neuorientierung unserer Gedanken über die Welt vorzunehmen. Genau darin liegt auch NRTs politische Kritik, eine Kritik an dem, was als Wissen gilt (Dewsbury, 2009:321). Eine Möglichkeit hierfür ist es sich von den Verbindungen eines Assemblages leiten zu lassen. Es geht dabei um ein aktives In-Verbindung-Setzen innerhalb eines Assemblages. Eine nicht-repräsentationale Herangehensweise lässt uns dann (Sperr-)Müll nicht mehr nur als inerte Materie erleben, die darauf wartet abgeholt zu werden, sondern als Situation, in der wir uns mit anderen Dingen, Körpern und Umwelten verbinden können. Sperrmüll kann so als eine Möglichkeit verstanden werden, wie Tom Roberts (2012:2526) es treffend schreibt, „to speculate upon the material capacities for being ,swept away" for the better. (...) [It] is an exploration for the potential to change, a concern for questioning the world's becoming-other".

\section{Datenverfügbarkeit}

Dieser Artikel basiert u.a. auf ethnographischer Forschung. Die Daten sind mit Ausnahme der beiden Tonschnipsel nicht öffentlich zugänglich. Diese finden Sie unter:

- doi:10.5446/20233

- doi:10.5446/20233

\section{Haftungsausschluss}

Ich versichere eidesstattlich, die vorliegende Arbeit selbständig verfasst und keine anderen als die angegebenen Quellen benutzt zu haben. Alle wörtlichen und sinngemäßen Entlehnungen sind unter genauer Angabe der Quelle kenntlich gemacht.

Danksagung. Ich danke Madlen Hornung, Martin Weigelt, Tom Keating und Mark Bauer für ihre unablässige Unterstützung und ihre guten Ideen. Weiterhin danke ich Matthew Hannah sowie den anonymen Gutachter_innen und Editor_innen für ihre hilfreiche, konstruktive Kritik an früheren Textversionen. Das verwendete Bildmaterial wurde von mir aufgenommen.

Edited by: A. Otto

Reviewed by: two anonymous referees

\section{Literatur}

Alliez, É. und Goffey, A.: Introduction, in: The Guattari effect, Herausgeber: Alliez, É. und Goffey, A., 8-14, Continuum, London, 2011.

Anderson, B. und Harrison, P.: The promise of non-representational theories, in: Taking-place: non-representational theories and geography, Herausgeber: Anderson, B. und Harrison, P., 1-34, Ashgate, Farnham, 2010.

Anderson, B. und McFarlane, C.: Assemblage and geography, Area, 43, 124-127, 2011.

Bassett, K.: Walking as an aesthetic practice and a critical tool: some psychogeographic experiments, J. Geogr. Higher. Educ., 28, 397-410, 2004.

Bates, C.: Video diaries: audio-visual research methods and the elusive body, Vis. Stud., 28, 29-37, 2013.

Berlina, B.: Raum, Überwachung, Kontrolle: vom staatlichen $\mathrm{Zu}$ griff auf städtische Bevölkerung, Westfällisches Dampfboot, Münster, 2006.

Benjamin, W.: The arcades project, Belknap Press, Cambridge, 2002.

Bennett, J.: Vibrant matter. A political ecology of things, Duke University Press, Durham, 2010.

Buchanan, I.: Deleuze and Guattari's Anti-Oedipus, Continnum, London, 2008.

Colebrook, C.: Gilles Deleuze, Routledge, London, 2002.

Colebrook, C.: Death of the posthuman: essays on extinction Vol. 1, Open Humanity Press, Michigan, [online] available at: http: //quod.lib.umich.edu/o/ohp/12329362.0001.001 (last access: 7 August 2016), 2014. 
Connolly, W. E.: Method, problem, faith, in: Problems and methods in the study of politics, Herausgeber: Shapiro, I., Smith, R. M., und Masoud, T. E., 332-349, Cambridge University Press, Cambridge, 2004.

Cortázar, J.: Teufelsgeifer, in: Urlaubslesebuch, Herausgeber: Adler, K., 90-109, Dt. Taschenbuch-Verlag, München, 2010.

Davies, G. und Dwyer, C.: Qualitative methods: are you enchanted or are you alienated?, Prog. Hum. Geogr., 31, 257-266, 2007.

Debord, G.: Theorie des Umherschweifens, Situationistische Internationale, 58(2), o.S., [online] aufrufbar: http://www.si-revue.de/ theorie-des-umherschweifens (letzter Zugriff: 20 October 2016), 1958.

de Certeau, M.: Kunst des Handelns, Merve Verlag, Berlin, 1988.

Deleuze, G.: Bergsonism, Zone Books, New York, 1988a.

Deleuze, G.: Spinoza: practical philosophy, City Lights Books, San Francisco, 1988b.

Deleuze, G.: Unterhandlungen: 1972-1990, Suhrkamp, Frankfurt am Main, 1993.

Deleuze, G. und Guattari, F.: Kafka: für eine kleine Literatur, Suhrkamp, Frankfurt am Main, 1976.

Deleuze, G. und Guattari, F.: Anti-Ödipus: Kapitalismus und Schizophrenie I, Suhrkamp, 1977.

Deleuze, G. und Guattari, F.: Tausend Plateaus: Kapitalismus und Schizophrenie II, Merve Verlag, Berlin, 1992.

Deleuze, G. und Guattari, F.: Was ist Philosophie?, Suhrkamp, Frankfurt am Main, 1996.

DeLyser, D.: Writing qualitative geography, in: The SAGE handbook of qualitative geography, Herausgeber: DeLyser, D., Herbert, S., Aitken, S., Crang, M. und McDowell, L., 341-358, SAGE Publications, London, 2009.

DeSilvey, C.: Observed Decay: Telling Stories with Mutable Things, J. Mat. Cult., 11, 318-338, 2006.

Dewsbury, J.-D.: Performative, non-representational, and affectbased research: seven injunctions, in: The SAGE handbook of qualitative geography, Herausgeber: DeLyser, D., Herbert, S., Aitken, S., Crang, M., und McDowell, L., 321-334, SAGE Publications, London, 2009.

Dewsbury, J.-D.: Language and the event: the unthought of appearing worlds, in: Taking-place: non-representational theories and geography, Herausgeber: Anderson, B. und Harrison, P., 147160, Ashgate, Farnham, 2010.

Dewsbury, J.-D.: The Deleuze-Guattarian assemblage: plastic habits, Area, 43, 148-153, 2011a.

Dewsbury, J.-D.: The singularity of the ,still“: „,never suspend the question“, in: Stillness in a mobile world, Herausgeber: Bissell, D. und Fuller, G., 175-191, Routledge, London, 2011 b.

Dewsbury, J.-D.: Affective habit ecologies: material dispositions and immanent inhabitations, Perform. Res., 17, 74-82, 2012.

Dewsbury, J.-D.: Inscribing thoughts: the animation of an adventure, Cult. Geogr., 21, 147-152, 2014.

Dewsbury, J.-D. und Naylor, S.: Practising geographical knowledge: fields, bodies and dissemination, Area, 34, 253-260, 2002.

Dewsbury, J.-D., Harrison, P., Rose, M. und Wylie, J.: Enacting geographies, Geoforum, 33, 437-440, 2002.

Dirksmeier, P. und Helbrecht, I.: Time, non-representational theory and the ,performative turn“: towards a new methodology in qualitative social research, Forum Qualitative Sozialforschung/Forum: Qualitative Social Research, 9(2), 2008.
Dirksmeier, P. and Helbrecht, I.: Intercultural interaction and "situational places": a perspective for urban cultural geography within and beyond the performative turn, Soc. Geogr., 5, 39-48, doi:10.5194/sg-5-39-2010, 2010.

Dirksmeier, P. und Helbrecht, I.: Die Beobachtung der Situation: Zur Rolle von Affekten in Begenung zwischen Fremden, Geogr Z., 101, 65-81, 2013.

Doel, M. A.: Representation and difference, in: Taking-place: nonrepresentational theories and geography, Herausgeber: Anderson, B. und Harrison, P., 117-130, Ashgate, Farnham, 2010.

Edensor, T.: Waste matter: the debris of industrial ruins and the disordering of the material world, J. Mater. Cult., 10, 311-332, 2005.

Federman, R.: Take it or leave it: an exaggerated second-hand tale to be read aloud either standing or sitting, Fiction Collective, New York, NY, 1976.

Foer, J. S.: Extremely loud \& incredibly close, Penguin, London, 2006.

Gabrys, J.: Sink: the dirt of systems, Environ. Plan. Soc. Space, 27, 666-681, 2009.

Gabrys, J., Hawkins, G. und Michael, M.: Introduction: from materiality to plasticity, in: Accumulation the material politics of plastic, Herausgeber: Gabrys, J., Hawkins, G. und Michael, M., 1-14, Routledge, Abingdon, 2013.

Geertz, C.: Dichte Beschreibung: Beiträge zum Verstehen kultureller Systeme, Suhrkamp, Frankfurt am Main, 1983.

Graham, S. und Thrift, N.: Out of order: understanding repair and maintenance, Theory Cult. Soc., 24, 1-25, 2007.

Gregson, N. und Crang, M.: Materiality and waste: inorganic vitality in a networked world, Environ. Plan. A, 42, 1026-1032, 2010.

Gregson, N., Metcalfe, A., und Crewe, L.: Identity, mobility, and the throwaway society, Environ. Plan. Soc. Space, 25, 682-700, 2007.

Halsey, M.: Ecology and machinic thought, Angelaki, 10, 33-55, 2005.

Hannah, M. G.: Innovations in the afterlife of the Cold War: German-language human geography, Soc. Cult. Geogr., 17, 71$80,2016$.

Hawkins, G.: The ethics of waste: how we relate to rubbish, Rowman \& Littlefield Publishers, Lanham, 2006.

Henare, A. J. M., Holbraad, M. und Wastell, S.: Introduction: thinking through thinks, in: Thinking through things, Herausgeber: Henare, A. J. M., Holbraad, M. und Wastell, S., 1-31, UCL, London, 2007.

Hesse, M.: Retheorizing urban geography: mutual relationships between English- and German-speaking communities, Soc. Cult. Geogr., 17, 52-70, 2016.

Hutta, J. S.: The affective life of semiotics, Geogr. Helv., 70, 295 309, doi:10.5194/gh-70-295-2015, 2015.

Jenks, C. und Neves, T.: A walk on the wild side: urban ethnography meets the Flâneur, Cult. Values, 4, 1-17, 2000.

Jones, P. und Evans, J.: Rescue Geography: Place Making, Affect and Regeneration, Urban Stud., 49, 2315-2330, 2012.

Jöns, H. und Freytag, T.: Boundary spanning in social and cultural geography, Soc. Cult. Geogr., 17, 1-22, 2016.

Kazig, R.: Atmosphären. Konzept für einen nicht repräsentationellen Zugang zum Raum, in: Kulturelle Geographien: Zur Beschäftigung mit Raum und Ort nach dem Cultural Turn, Herausgeber: Berndt, C. und Pütz, R., 167-187, Transcript, Bielefeld, 2007. 
Korf, B.: Geographie und Gehirn, Berichte Zur Dtsch. Landeskd., 81, 267-284, 2007.

Korf, B.: Neuro-Kulturgeographie, Geogr. Z., 100, 146-163, 2012.

Lash, S.: Life (Vitalism), Theor. Cult. Soc., 23, 323-329, 2006.

Latham, A. und McCormack, D. P.: Thinking with images in nonrepresentational cities: vignettes from Berlin, Area, 41, 252-262, 2009.

Law, J.: After method: mess in social science research, Routledge, London, 2004.

Lefebvre, H.: Critique of everyday life, Verso, London, 1991.

Lorimer, H.: Cultural geography: the busyness of being "more-thanrepresentational“", Prog. Hum. Geog., 29, 83-94, 2005.

Lorimer, H.: Afterword: non-representational theory and me too, in: Non-representational methodologies, Re-envisioning Research, Herausgeber: Vannini, P., 177-187, Routledge, London, 2015.

Lorimer, J.: Moving image methodologies for more-than-human geographies, Cult. Geogr., 17, 237-258, 2010.

Lossau, J.: „Mind the gap“: Bemerkungen zur gegenwärtigen Raumkonjunktur aus kulturgeographischer Sicht, in: Topologie: Zur Raumbeschreibung in den Kultur- und Medienwissenschaften, Herausgeber: Günzel, S., 53-68, Transcript, Bielefeld, 2007.

MacFarlane, R.: A Road of one's own: past and present artists of randomly motivated walk, Times Lit. Suppl., 7, 3-4, 2005.

Mattissek, A. and Wiertz, T.: Materialität und Macht im Spiegel der Assemblage-Theorie: Erkundungen am Beispiel der Waldpolitik in Thailand, Geogr. Helv., 69, 157-169, doi:10.5194/gh-69-1572014, 2014.

McFarlane, C.: The city as assemblage: dwelling and urban space, Environ. Plan. Soc. Space, 29, 649-671, 2011.

Merriman, P.: Driving places: Marc Augé, non-places, and the geographies of England's M1 motorway, in: Automobilities, Herausgeber: Featherstone, M., Thrift, N. und Urry, J., 145-169, SAGE Publications, London., 2005.

Miggelbrink, J.: Diskurs, Machttechnik, Assemblage, Geogr. Z. Neue Impulse Für Eine Reg. Forsch., 102, 25-40, 2014.

Militz, E. und Schurr, C.: Affective nationalism: Banalities of belonging in Azerbaijan, Polit. Geogr., [online] available at: http: //linkinghub.elsevier.com/retrieve/pii/S0962629815000967 (last access: 24 August 2016), 2015.

Miller, D.: The comfort of things, Polity, Cambridge, 2008.

Miller, J. C.: Malls without stores (MwS): the affectual spaces of a Buenos Aires shopping mall, Trans. Inst. Br. Geogr., 1-12, 2012.

Mol, A.: Ontological politics: a word and some questions, in: Actor Network Theory and After, Herausgeber: Law, J. und Hassard, J., 74-89, Blackwell, Oxford, 1999.

Moore, S. A.: Global garbage: waste, trash trading, and local garbage politics, in: Global political ecology, Herausgeber: Peet, R., Robbins, P. und Watts, M., 133-143, Routledge, London, New York, 2011.

Moore, S. A.: Garbage matters: concepts in new geographies of waste, Prog. Hum. Geog., 36, 780-799, 2012.

Müller, M.: More-than-representational political geographies, in: The Wiley-Blackweel companion to political geography, Herausgeber: Agnew, J., Mamadouh, V., Secor, A. und Sharp, J., 409422, Wiley-Blackwell, Oxford, 2015.

Nancy, J.-L.: God, Justice, Love, Beauty: four little dialogues, Fordham University Press, New York, 2011.

Obersteiner, G.: Introduction to the project "TransWaste", http: //www.transwaste.eu/file/001328.pdf, 23 July 2014, 2010.
Obersteiner, G., Linzer, R., Pertl, A., Scherhaufer, S. und Schmied, E.: Formalisation of informal sector activities in collection and transboundary shipment of waste in and to CEE: introduction to the project "Transwaste", http://www.transwaste.eu/file/001444. pdf, 23 July 2014, 2010.

Oldrup, H. H. und Carstensen, T. A.: Producing geographical knowledge through visual methods, Geogr. Ann. B, 94, 223-237, 2012.

Peet, R., Robbins, P. und Watts, M.: Global nature, in: Global political ecology, Herausgeber: Peet, R., Robbins, P. und Watts, M. 1-47, Routledge, London, New York, 2011.

Perec, G.: Species of spaces and other pieces, Penguin, London, 2008.

Perec, G.: Träume von Räumen, Diaphanes, Zürich., 2013.

Pikner, T. und Jauhiainen, J. S.: Dis/appearing waste and afterwards, Geoforum, 54, 39-48, 2014.

Reed-Danahay, D.: Introduction, in: Auto/ethnography: rewriting the self and the social, Herausgeber: Reed-Danahay, D., 1-17, Berg, Oxford, 1997.

Richardson-Ngwenya, P.: Performing a more-than-human material imagination during fieldwork: muddy boots, diarizing and putting vitalism on video, Cult. Geogr., 21, 293-299, 2014.

Robbins, P. und Marks, B.: Assemblage geographies, in: The Sage handbook of social geographies, Herausgeber: Smith, S. J., Pain, R., Jones III, J. P. und Marston, S. A., 176-194, SAGE Publications, Thousand Oaks, 2009.

Roberts, T.: From "new materialism" to "machinic assemblage": agency and affect in IKEA, Environ. Plan. A, 44, 2512-2529, 2012.

Rogers, H.: Gone tomorrow: the hidden life of garbage, New Press, New York, 2005.

Saunders, A.: Recovering the street: relocalising urban geography, J. Geogr. Higher Educ., 37, 536-546, 2013.

Schurr, C.: Emotionen, Affekte und nicht-repräsentationale Geographie, Geogr. Z., 102, 148-161, 2014.

Schurr, C. and Strüver, A.: „The Rest“: Geographien des Alltäglichen zwischen Affekt, Emotion und Repräsentation, Geogr. Helv., 71, 87-97, doi:10.5194/gh-71-87-2016, 2016.

Simpson, P.: 'So, as you can see ... ': some reflections on the utility of video methodologies in the study of embodied practices, Area, 43, 343-352, 2011.

Simpson, P.: Apprehending everyday rhythms: rhythmanalysis, time-lapse photography, and the space-times of street performance, Cult. Geogr., 19, 423-445, 2012.

Simpson, P.: Ecologies of experience: materiality, sociality, and the embodied experience of (street) performing, Environ. Plan. A, 45, 180-196, 2013.

Smith, N. und Low, S.: Introduction: the imperative of public space, in The politics of public space, Herausgeber: Low, S. und Smith, N., 1-16, Routledge, New York, 2006.

Strüver, A.: Der Konstruktivismus lernt laufen: Doing more-thanrepresentational geography, Soc. Geogr., 6, 1-13, 2011.

Thompson, R. C.: Plastic, environment and human health, in: Accumulation the material politics of plastic, Herausgeber: Hawkins, G. und Michael, M., 150-167, Routledge, Abingdon, 2013.

Thrift, N.: Summoning Life, in: Envisioning human geographies, Herausgeber: Cloke, P. J., Crang, P. und Goodwin, M., 81-103, Arnold, London, 2004. 
Thrift, N.: Non-representational theory: space, politics, affect, Routledge, London, 2008.

Thrift, N. und Dewsbury, J.-D.: Dead geographies - and how to make them live, Environ. Plan. Soc. Space, 18, 411-432, 2000.

Vannini, P.: Non-representational research methodologies: an introduction, in: Non-representational methodologies: re-envisioning research, Herausgeber: Vannini, P., 1-18, Routledge, London, 2015.

Waitt, G., Ryan, E. und Farbotko, C.: A visceral politics of sound, Antipode, 46, 283-300, 2014.

WDR: Sperrmüll: save the waste - Goldgrube Sperrmüll, [online] available at: http://www1.wdr.de/fernsehen/ratgeber/ haushaltscheck/sendungen/uebersichtsperrmuell100.html, last access: 8 August 2014.

WDR 5: Eine Woche nach dem Unwetter - WDR 5, [online] available at: http://www.wdr5.de/sendungen/morgenecho/ kampfgegenpluenderer114.html, last access: 8 August 2014.
WienerZeitung: Legalität für Müllsammler, [online] available at: http://www.wienerzeitung.at/nachrichten/oesterreich/chronik/ 505732_Legalitaet-fuer-Muellsammler.html (last access: 8 August 2014), 2012.

Wrights \& Sites: A manifesto for a new walking culture: "dealing with the city“, Perform. Res., 11, 115-122, 2006.

Wylie, J.: An essay on ascending Glastonbury Tor, Geoforum, 33, 441-454, 2002.

Wylie, J.: A single day's walking: narrating self and landscape on the South West Coast Path, Trans. Inst. Br. Geogr., 30, 234-247, 2005.

Yi'En, C.: Telling stories of the city: walking ethnography, affective materialities, and mobile encounters, Space Cult., 2013.

Żurańska-Skalny, M.: Informal collection and marketing of waste/products in Lower Silesia (Poland), [online] available at: http: //www.transwaste.eu/file/001554.pdf (last access: 26 July 2015), 2010. 